UWThPh-1996-40

\title{
Nonperturbative Stochastic Quantization of the Helix Model
}

\author{
Helmuth Hüffel* and Gerald Kelnhofer** \\ Institut für Theoretische Physik \\ Universität Wien \\ Boltzmanngasse 5, A-1090 Vienna, Austria
}

\begin{abstract}
The helix model describes the minimal coupling of an abelian gauge field with three bosonic matter fields in $0+1$ dimensions; it is a model without a global Gribov obstruction. We perform the stochastic quantization in configuration space and prove nonperturbatively equivalence with the path integral formalism. Major points of our approach are the geometrical understanding of separations into gauge independent and gauge dependent degrees of freedom as well as a generalization of the stochastic gauge fixing procedure which allows to extract the equilibrium Fokker-Planck probability distribution of the model.
\end{abstract}

*) email: helmuth.hueffel@univie.ac.at

**) supported by "Fonds zur Förderung der wissenschaftlichen Forschung in Österreich", project P10509-NAW 


\section{Introduction}

An alternative scheme for quantization based on stochastic averages has been presented several years ago by Parisi and Wu [1], see refs. [2, [4] for comprehensive reviews and referencing. The main idea of "stochastic quantization" in configuration space - in this paper we will exclusively discuss the configuration space quantization - is to view Euclidean quantum (field) theory as the equilibrium limit of a statistical system coupled to a thermal reservoir. This system evolves in a new additional time direction which is called stochastic time until it reaches the equilibrium limit for infinite stochastic time. The coupling to the heat reservoir is simulated by means of a stochastic noise field - or rather by the mathematically well defined Wiener process - which forces the original Euclidean field to wander randomly. In the equilibrium limit the stochastic averages become identical to ordinary Euclidean vacuum expectation values.

There are two equivalent formulations of stochastic quantization due to the general properties of stochastic processes: In one formulation all fields have an additional dependence on stochastic time. Their stochastic time evolution is determined by a Langevin equation which has a drift term constructed from the gradient of the classical action of the system. The expectation values of observables are obtained by ensemble averages over the Wiener measure.

Corresponding to this Langevin equation is an equivalent diffusion process which is defined in terms of a Fokker Planck equation for the probability distribution characterizing the stochastic evolution of the system. Now expectation values of the observables are defined by functionally integrating over them with the stochastic time dependent Fokker-Planck probability distribution.

In several non-gauge models (see especially [4] for a mathematically rigorous treatment of scalar field theory) one can verify that in the infinite stochastic time limit this FokkerPlanck probability distribution converges to the standard Euclidean configuration space path integral density.

Over the past years the stochastic quantization scheme has grown into a useful tool in 
several areas of quantum field theory and a large number of new results and generalized schemes have appeared [5, 6]: In specific we would like to recall the applications to gauge fields (see below), fermionic fields, the studies of anomalies, investigations of supersymmetric models and the regularization and renormalization program; last but not the least the numerical simulations became an important non perturbative application of stochastic quantization; stochastic techniques could be applied to Monte-Carlo simulations by means of the so called hybrid algorithms.

One might attach great importance especially to the numerical applications of the stochastic quantization scheme while supposing that its formal field theoretic developments have been completed. It is our conviction, however, that the method of stochastic quantization can be distinguished by its fundamental concepts and therefore seems still relevant for actual research. In this connection we refer to the quantization of gauge theories:

In configuration space the most prominent quantization scheme is provided by the path integral using the Faddeev-Popov procedure [7], which guarantees the unitarity of the corresponding Feynman amplitudes. However, due to the Gribov ambiguity 8, 9], which is related to the fact that the topological nontrivial structure of the space of gauge orbits prevents the existence of a global gauge fixing, the path integral quantization is incomplete beyond the perturbation theory. On the perturbative level this does not lead to difficulties since one stays within the local region where the gauge fixing is well defined. There have been many attempts to deal with these nonperturbative aspects in the path integral [10, 11, 12, 14, 13] but a profound solution has not been found yet.

Much hope has been put forward to gain new insights for a correct nonperturbative path integral formulation of gauge theories from the stochastic quantization point of view [15, 16]. The stochastic quantization scheme of gauge theories in configuration space has been developed apart of the above dual structure of a Langevin and Fokker-Planck formulation in two related ways:

a) Parisi and $\mathrm{Wu}$ [1] proposed the quantization in terms of Langevin equations without gauge fixing terms and without Faddeev-Popov ghosts. Since the pure action 
of the gauge model remains invariant under gauge transformations, the associated drift term of the Langevin equation acts orthogonally to the gauge orbits. As a consequence unbounded diffusion along the orbits takes place so that potential divergencies are arising for the expectation values of general observables. It is argued, however, that these contributions are canceling out among themselves in expectation values of gauge invariant observables.

This Parisi-Wu stochastic quantization method of gauge theories preserves the full gauge symmetry and provides a manifestly gauge covariant kind of quantization. Due the absence of gauge fixing it was claimed to be valid also in the nonperturbative regime without the Gribov ambiguity.

b) In the approach of Zwanziger [17] the drift term of the stochastic process is modified by the addition of a gauge fixing force tangent to the gauge orbits. It provides damping for the gauge modes' diffusion along the orbits leaving, however, unchanged all gauge invariant expectation values. This "stochastic gauge fixing" procedure allows to directly resort to a well defined Fokker-Planck formulation so that powerful functional techniques can be applied. Again no Faddev-Popov ghosts are needed and no Gribov ambiguity was claimed to exist neither.

A fundamental question arises how stochastic quantization - if at all - compares with the conventional quantization schemes in the case of gauge theories. A great amount of work shows agreement at the perturbative level up to some order in the coupling constants, e.g. for Yang-Mills theory up to two loops [18]. The question of equivalence, however, becomes subtle at the perturbative level to any order and turns into a hot subject at the nonperturbative level.

Unfortunately - due to the unbounded diffusion along the gauge orbits - the Parisi -Wu scheme for gauge theories lacks an immediate Fokker-Planck formulation and equilibrium properties are hard to extract. This comes from the technical difficulty to solve exactly the corresponding Langevin equations; no general equivalence proofs of any nontrivial gauge model have been established. 
On the other hand using Zwanziger's scheme it is necessary to

a) rigorously control the required stabilizing effect along the gauge orbits

b) prove convergence to equilibrium of the Fokker-Planck probability distribution

c) explicitly find out what this limit is.

With respect to these requirements according to our opinion again no satisfactory nonperturbative treatment of any nontrivial gauge model has been given (see however the formal procedures [19, 20]).

In view of the above difficulties it is not surprising that the interest in the stochastic quantization scheme - despite of its conceptional beauties - has gone down remarkably over the last years .

It seems first of all helpful to choose a gauge model which is as simple as possible, containing, however, relevant physical and mathematical structure.

From a pure mathematical viewpoint, a gauge model coupled to matter fields can be formulated in terms of fiber bundle theory. The fields form an infinite dimensional configuration space on which an action of the symmetry group of the model is given. Reducing to the true degrees of freedom corresponds to projecting the theory down to the true configuration space, which is obtained by taking the quotient of the configuration space by the gauge group. Depending on the kind of action of the gauge group this quotient may have a smooth or an orbifold structure.

The problem of the orbifold structure of the physical phase space has been addressed by several authors (see e.g. [23, 22, 21]). We remind, however, that in this paper we are restricting ourselves to just a configuration space discussion. In this respect we would like to quote relations with inequivalent quantizations in two dimensional Yang-Mills theory on a cylinder by [24, 25] and the analysis of the singularity structure of the Yang-Mills configuration space by [26].

In order to gain new insights into the stochastic quantization scheme of gauge theories it seems important to analyze how these geometrical concepts are encoded in the 
corresponding Langevin or Fokker-Planck equation, respectively. More precisely, we want to study the evolution properties of the stochastic process separately along the true configuration space as well as along the gauge group. In order to avoid orbifold singularities and Gribov ambiguities it is appropriate to study such a gauge model in which

i) the gauge group acts freely, that is without fixed points.

ii) the separation into gauge independent and gauge dependent degrees of freedom is globally possible.

It is well known (see e.g. [27]) that under these circumstances the true configuration space is a smooth manifold. Technically, this separation corresponds to the choice of a particular trivialization of the principal fiber bundle on which the stochastic process is formulated.

For these reasons we decided to choose the so called Helix model, which describes the minimal coupling of an abelian gauge field with three bosonic matter fields in $0+$ 1 dimensions. This model was originally proposed by deWit [28] and was investigated intensively within the Hamiltonian framework by Kuchar [29]. Recently the helix model came to new life again [13] in the course of studies on problems with gauge fixing and the Gribov ambiguity; it was further analyzed within the BRST approach by [14].

To clarify we remark that although globally the helix model is free from topological gauge fixing obstructions one may nevertheless find specific gauge choices which suffer from Gribov ambiguities. The helix model therefore serves as an ideal testing ground for treatments of "bad" gauge choices and their associated Gribov problems.

In the present paper (see also [30, 31]) we perform the stochastic quantization procedure by exploiting the globally unproblematic structure of the helix model. We will come back to the discussion of the above - artificial - complications of the model in a separate publication; there we will be analyzing gauge choices where one is forced to work on coordinate patches [32.

We present in this paper a refined stochastic gauge fixing procedure and prove nonperturbatively the equivalence of the stochastically quantized helix model to the known 
path integral formulation. We propose in fact a modified stochastic process where not only the drift term is changed but also the Wiener process itself. These modifications are done in such a way that a proper damping of the gauge modes along the orbits is possible, that all gauge invariant expectation values remain unchanged and that the equilibrium Fokker-Planck distribution can be determined; it is seen to agree with the known path integral density of the helix model [13].

Although our generalized scheme of stochastic gauge fixing is worked out here for the helix model we will give arguments for its general applicability.

We start in section 2) with a review on basic facts of Ito's stochastic calculus and recall in section 3) essentials of the Parisi-Wu stochastic quantization scheme of non-gauge models.

A discussion on the geometric structure of the helix model in configuration space is given in section 4).

In section 5) we present an overview of the stochastic quantization of gauge models and derive our generalization of the stochastic gauge fixing procedure.

The damping of the gauge degrees of freedom is discussed in section 6).

In section 7) we derive the equilibrium Fokker-Planck distribution of the helix model in a nonperturbative manner and show that is agreeing with the known path integral density of this model.

Section 8) deals with geometrical aspects of the generalized stochastic gauge fixing scheme.

We present an outlook in section 9) and add a short appendix for technical details.

\section{A short review of the Ito Stochastic Calculus}

In this section we review basic facts of the Ito stochastic calculus (see e.g. 33], 34, 35]) assuming that the reader is already familiar with the concepts of stochastic processes.

A set of Langevin equations for $\mathrm{m}$ scalar fields $\Phi^{i}(t, s), i=1, . ., m$, which depend on a real coordinate $t$ (which in the following sections will be interpreted as Euclidean time) 
and the stochastic time coordinate $s$

$$
d \Phi^{i}(t, s)=a^{i}(t, s) d s+\int_{\mathbf{R}} d t^{\prime} b^{i}{ }_{k}\left(t, t^{\prime} ; s\right) d W^{k}\left(t^{\prime}, s\right)
$$

is an abreviation for the associated set of stochastic integral equations

$$
\Phi^{i}(t, s)=\int_{s_{0}}^{s} a^{i}(t, s) d \sigma+\int_{\mathbf{R}} d t^{\prime} \int_{s_{0}}^{s} b^{i}{ }_{k}\left(t, t^{\prime} ; \sigma\right) d W^{k}\left(t^{\prime}, \sigma\right) .
$$

The $a^{i}$ and the $b^{i}{ }_{k}$ are given functions of the fields $\Phi^{i}$, which are defined at just the same stochastic time $s$. As a consequence the $a^{i}$ and the $b^{i}{ }_{k}$ are local in the stochastic time $s$ but are allowed to generally be nonlocal in the other variable $t$. Later in our paper we will in fact discuss cases where the $a^{i}$ and the $b^{i}{ }_{k}$ imply differentiations and integrations of the $\Phi^{i}$ with respect to $t$.

$d W^{i}(t, s)$ denote the increments of an $m$-dimensional Wiener process $W^{i}(t, s)$, which have the correlations

$$
\left\langle\left\langle d W^{i}(t, s) d W^{k}\left(t^{\prime}, s\right)\right\rangle\right\rangle=2 \delta^{i k} \delta\left(t-t^{\prime}\right) d s .
$$

The second integral in (2.2) is a stochastic integral; it is defined in the mean square sense as the limit of an infinite Riemann-Stieltjes sum, which, however, carries a dependence on the choice of the positions of the intermediate points. We adopt Ito's choice to define the integral according to the product rule

$$
b_{k}^{i}\left(t, t^{\prime} ; s\right) d W^{k}\left(t^{\prime}, s\right)=\lim _{\Delta s \rightarrow 0} b^{i}{ }_{k}\left(t, t^{\prime} ; s\right)\left[W^{k}(t, s+\Delta s)-W^{k}(t, s)\right]
$$

and call the corresponding stochastic differential equation (2.1) an Ito-Langevin equation.

Under certain conditions (Lippschitz condition, growth condition, specific class of initial conditions) it can be proven that the solution of the Ito-Langevin equation (2.1) is a unique nonanticipating function. A function $g(s)$ is called non-anticipating if for $s<s^{\prime}$ it is statistically independent of $W(s)-W\left(s^{\prime}\right)$.

Expectation values of arbitrary functions of the solutions $\Phi^{i}(t, s)$ are obtained by ensemble averages over the Wiener measure, using the Gaussian behaviour of the Wiener process and applying repeatedly the correlations (2.3). 
One consequence of Ito's choice is the mean value formula

$$
\left\langle\left\langle\int_{s_{0}}^{s} f(\Phi(t, \sigma)) d W^{i}(t, \sigma)\right\rangle\right\rangle=0
$$

which follows from the non-anticipating nature of the solutions of the Ito-Langevin equation; $f$ is an arbitrary (non-anticipating) function of $\Phi^{i}$.

Next we recall the so called Ito formula, which states that the differential of an arbitrary function(al) $f$ of $\Phi^{i}$ is given by

$$
\begin{aligned}
d f[\Phi]= & \int_{\mathbf{R}} d t_{1}\left\{\left[\frac{\delta f}{\delta \Phi^{i}\left(t_{1}\right)} a^{i}\left(t_{1}, s\right)+\int_{\mathbf{R}} d t_{2} d t_{3} \frac{\delta^{2} f}{\delta \Phi^{i}\left(t_{1}\right) \delta \Phi^{k}\left(t_{2}\right)} b_{j}^{i}\left(t_{1}, t_{3} ; s\right) \delta^{j \ell} b^{k}{ }_{\ell}\left(t_{2}, t_{3} ; s\right)\right] d s\right. \\
& \left.+\int_{\mathbf{R}} d t_{2} \frac{\delta f}{\delta \Phi^{i}\left(t_{2}\right)} b_{k}^{i}\left(t_{1}, t_{2} ; s\right) d W^{k}\left(t_{2}, s\right)\right\}\left.\right|_{\Phi(\cdot)=\Phi(\cdot, s)}
\end{aligned}
$$

Here it is assumed that $f$ is twice partially continuosly differentiable with respect to $\Phi^{i}$.

Equivalently to (2.6) we can formally define multiplication rules for the differentials $d W^{i}$

$$
\begin{aligned}
d W^{i}(t, s) d W^{k}\left(t^{\prime}, s\right) & =2 \delta^{i k} \delta\left(t-t^{\prime}\right) d s \\
d W^{i}(t, s)^{2+N} & =0 \quad N=1,2, \ldots \\
d W^{i}(t, s) d s & =0
\end{aligned}
$$

which - more precisely - are given in terms of associated integrals over arbitrary nonanticipating functions. From (2.6) or (2.7) amounts, unfortunately, the cumbersome conclusion that in calculating expressions involving infinitesimals $d s$ of the stochastic time one must keep all expansions up to the second order in the $d W^{i}$.

What usually is meant by Ito's stochastic calculus can be summarized by equations $(2.4)-(2.7)$.

It is of fundamental significance that with the help of Ito's as well as the mean value formula one can derive from the Ito-Langevin equation (2.1) the Fokker-Planck equation for a (conditional) probability density $\rho$

$$
\frac{\partial \rho[\Phi, s]}{\partial s}=L[\Phi] \rho[\Phi, s] .
$$


Here the so called Fokker-Planck operator $L$ is defined in terms of the $a^{i}$ and the $b^{i}{ }_{k}$ as

$$
L[\Phi]=\int_{\mathbf{R}} d t_{1} \frac{\delta}{\delta \Phi^{i}\left(t_{1}\right)}\left[-a^{i}\left(t_{1}\right)+\int_{\mathbf{R}^{2}} d t_{2} d t_{3} \frac{\delta}{\delta \Phi^{k}\left(t_{2}\right)}\left(b_{j}^{i}\left(t_{1}, t_{3}\right) \delta^{j \ell} b_{\ell}^{k}\left(t_{2}, t_{3}\right)\right)\right] .
$$

It should be stressed that in a Fokker-Planck formulation the fields $\Phi^{i}$ - and consequently also the $a^{i}$ and the $b^{i}{ }_{k}$ - are stochastic time independent; all the stochastic time dependence is coming from the probability density $\rho$ itself. We therefore omit to denote a $s$-dependence of $\Phi^{i}$, as well as of $a^{i}$ and $b^{i}{ }_{k}$.

Due to the fundamental relationship between the Ito-Langevin (2.1) and the FokkerPlanck $(2.8,2.9)$ scheme the expectation values of arbitrary (equal stochastic time) functions $f$ of the solutions $\Phi^{i}(t, s)$ of the Ito-Langevin equation are coinciding with expectation values defined correspondingly in terms of the Fokker-Planck density $\rho$

$$
\langle\langle f(\Phi(\cdot, s))\rangle\rangle=\langle f(\Phi(\cdot))\rangle(s)=\int D \Phi f(\Phi(\cdot)) \rho[\Phi, s] .
$$

We remark again that in (2.10) there are involved stochastic time dependent as well as stochastic time independent fields, respectively.

The Fokker-Planck operator $L$ describes the stochastic time evolution of the FokkerPlanck probability density; with its help we formally write

$$
\rho[\Phi, s]=e^{s L[\Phi]} \rho[\Phi, s=0] .
$$

In case that we are concerned with the stochastic time evolution of expectation values, it is advantageous, however, to introduce in addition to $L$ also its adjoint $L^{\dagger}$

$$
\int D \Phi f(\Phi(\cdot)) \rho[\Phi, s]=\int D \Phi\left(e^{s L[\Phi]} \rho[\Phi, s=0]\right) f(\Phi(\cdot))=\int D \Phi \rho[\Phi, s=0] e^{s L^{\dagger}[\Phi]} f(\Phi(\cdot)) .
$$

It explicitly reads in terms of the $a^{i}$ and the $b^{i}{ }_{k}$

$$
L^{\dagger}[\Phi]=\int_{\mathbf{R}} d t_{1}\left[a^{i}\left(t_{1}\right)+\int_{\mathbf{R}^{2}} d t_{2} d t_{3} b^{i}{ }_{j}\left(t_{1}, t_{3}\right) \delta^{j \ell} b_{\ell}^{k}\left(t_{2}, t_{3}\right) \frac{\delta}{\delta \Phi^{k}\left(t_{2}\right)}\right] \frac{\delta}{\delta \Phi^{i}\left(t_{1}\right)} .
$$

At the end of this section we remark that we discussed Ito's stochastic calculus for fields $\Phi^{i}$ depending on just the coordinate(s) $t$ (and $s$ ), having in mind applications to the helix model. It would otherwise have been straightforward to generalize this section to a higher dimensional case as well. 


\section{A short review of the Parisi-Wu stochastic quan- tization scheme}

In this section we review some of the important features of the stochastic quantization scheme when performing the quantization of non-gauge models. We include this section for the benfit of the reader to get a self-contained presentation of our paper in order to be able to follow closely our subsequent discussions of the stochastic quantization procedure in the case of gauge theories.

We consider a model of $m$ (self-interacting) scalar fields $\Phi^{i}$ with the positive Euclidean action denoted by $S$. We assume that the model has a finite normalization integral $\int D \Phi e^{-S}$

The basic idea of the stochastic quantization scheme is to interpret the Euclidean path integral measure $e^{-S} / \int D \Phi e^{-S}$ as the equilibrium limit of a Fokker-Planck probability distribution of a specific stochastic process.

This stochastic process is defined by an Ito-Langevin equation (2.1) of the following form: The drift term $a^{i}$ is taken to be the variation of the action $S$ with respect to the fields $\Phi^{i}$, whereas the matrix $b^{i}{ }_{k}$ is chosen most simply as an $m$-dimensional unit matrix.

$$
d \Phi^{i}(t, s)=-\left.\delta^{i j} \frac{\delta S}{\delta \Phi^{j}(t)}\right|_{\Phi(t)=\Phi(t, s)} d s+d W^{i}(t, s) .
$$

It then follows from (2.9) that the associated Fokker-Planck operator is just

$$
L[\Phi]=\int_{\mathbf{R}} d t \frac{\delta}{\delta \Phi^{i}(t)} \delta^{i j}\left[\frac{\delta S}{\delta \Phi^{j}(t)}+\frac{\delta}{\delta \Phi^{j}(t)}\right] .
$$

We nonperturbatively deduce that the equilibrium limit of the Fokker-Planck distribution is formally given by $e^{-S} / \int D \Phi e^{-S}$. In this paper we do not discuss the complications due to the issue of renormalization, see, however [4] or [40].

As a consequence the equal stochastic time expectation values of arbitrary observables (calculated either within the Langevin or Fokker-Planck approach, (2.10)) relax in the infinite stochastic time limit to the corresponding quantum Green functions

$$
\lim _{s \rightarrow \infty}\langle\langle f(\Phi(\cdot, s))\rangle\rangle=\lim _{s \rightarrow \infty}\langle f(\Phi(\cdot))\rangle(s)=\langle f(\Phi(\cdot))\rangle .
$$


For later application we study now a bijective change of variables of the original fields $\Phi^{i}(t)$ to new fields $\Psi^{\mu}(t)$ [36, 37, 38]. With respect to this variable change we introduce vielbeins $E$ and their inverses $e$

$$
E^{\mu}{ }_{i}\left(t, t^{\prime}\right)=\frac{\delta \Psi^{\mu}(t)}{\delta \Phi^{i}\left(t^{\prime}\right)}, \quad e_{\mu}^{i}\left(t, t^{\prime}\right)=\frac{\delta \Phi^{i}(t)}{\delta \Psi^{\mu}\left(t^{\prime}\right)},
$$

as well as the induced inverse metric $G^{\mu \nu}$ and the determinant $G$

$$
\begin{aligned}
G^{\mu \nu}\left(t_{1}, t_{2}\right) & =\int_{\mathbf{R}} d t_{3} E^{\mu}{ }_{i}\left(t_{1}, t_{3}\right) \delta^{i j} E^{\nu}{ }_{j}\left(t_{2}, t_{3}\right) \\
G & =\operatorname{det} G_{\mu \nu}\left(t_{1}, t_{2}\right) .
\end{aligned}
$$

The vielbeins, the inverse metric $G^{\mu \nu}$ and the determinant $G$ are generally field dependent and are used either in the Fokker-Planck or in the Langevin equations; in the latter case we have to explicitly insert the additional $s$-dependence of the fields.

With the Ito formula (2.6) the transformed Langevin equations for the new variables $\Psi^{\mu}$ read

$$
d \Psi^{\mu}(t, s)=\left.\int_{\mathbf{R}} d t^{\prime}\left\{E^{\mu}{ }_{i}\left(t, t^{\prime}\right)\left[-\frac{\delta S}{\delta \Phi^{i}\left(t^{\prime}\right)} d s+d W^{i}\left(t^{\prime}, s\right)\right]+\frac{\delta E^{\mu}{ }_{i}\left(t, t^{\prime}\right)}{\delta \Phi^{i}\left(t^{\prime}\right)} d s\right\}\right|_{\Phi(\cdot)=\Phi(\cdot, s)}
$$

which we can recast into

$$
\begin{aligned}
d \Psi^{\mu}(t, s)= & \int_{\mathbf{R}} d t^{\prime}\left\{\left[-G^{\mu \nu}\left(t, t^{\prime}\right) \frac{\delta S}{\delta \Psi^{\nu}\left(t^{\prime}\right)}+\frac{1}{\sqrt{G}} \frac{\delta}{\delta \Psi^{\nu}\left(t^{\prime}\right)}\left(G^{\mu \nu}\left(t, t^{\prime}\right) \sqrt{G}\right)\right] d s\right. \\
& \left.+E^{\mu}{ }_{i}\left(t, t^{\prime}\right) d W^{i}\left(t^{\prime}, s\right)\right\}\left.\right|_{\Psi(\cdot)=\Psi(\cdot, s)} .
\end{aligned}
$$

In the last equation we made use of the fact that the covariant divergence of the vielbein $E$ is vanishing (we considered the transformations from flat field variables to curved ones) so that

$$
\int_{\mathbf{R}} d t\left[\frac{\delta E^{\mu}{ }_{i}\left(t, t^{\prime}\right)}{\delta \Psi^{\mu}(t)}+\frac{1}{\sqrt{G}} E^{\mu}{ }_{i}\left(t, t^{\prime}\right) \frac{\delta \sqrt{G}}{\delta \Psi^{\mu}(t)}\right]=0
$$

and

$$
\int_{\mathbf{R}} d t^{\prime} \frac{\delta E^{\mu}{ }_{i}\left(t, t^{\prime}\right)}{\delta \Phi^{i}\left(t^{\prime}\right)}=\int_{\mathbf{R}} d t^{\prime} \frac{1}{\sqrt{G}} \frac{\delta}{\delta \Psi^{\nu}\left(t^{\prime}\right)}\left(G^{\mu \nu}\left(t, t^{\prime}\right) \sqrt{G}\right) .
$$

We interpret (3.7) as a stochastic process taking place in the manifold parametrized by the metric $G_{\mu \nu}$, see e.g. [36]; we observe the presence of the so called "Ito term", which appears as a simple consequence of the Ito formula. 
We are interested in the transformed form of the Fokker-Planck equation, too. We have just to use (2.9) in the case of the new variables $\Psi^{\mu}$, starting from the transformed Langevin equation (3.7). We immediately obtain the Fokker-Planck operator in the new variables as

$$
L[\Psi]=\int_{\mathbf{R}^{2}} d t d t^{\prime} \frac{\delta}{\delta \Psi^{\mu}(t)} G^{\mu \nu}\left(t, t^{\prime}\right)\left[\frac{\delta S}{\delta \Psi^{\nu}\left(t^{\prime}\right)}-\frac{1}{\sqrt{G}} \frac{\delta \sqrt{G}}{\delta \Psi^{\nu}\left(t^{\prime}\right)}+\frac{\delta}{\delta \Psi^{\nu}\left(t^{\prime}\right)}\right] .
$$

The formal equilibrium distribution now reads

$$
\rho[\Psi]_{\text {equil. }}=\frac{\sqrt{G} e^{-S}}{\int D \Psi \sqrt{G} e^{-S}}
$$

and we observe the appearance of the familiar Jacobian factor $\sqrt{G}$ corresponding to the variable transformation $\Phi^{i}$ to $\Psi^{\mu}$.

Finally we comment on an equivalence relation of stochastic processes in the infinite stochastic time limit, which is usually called fluctuation-dissipation theorem, or sometimes kernel independence of the stochastic process (see e.g. 34] and 39]). More precisely we consider Ito-Langevin equations of the form

$$
\begin{aligned}
d \Psi^{\mu}(t, s)= & \int_{\mathbf{R}} d t^{\prime}\left\{\left[-G^{\mu \nu}\left(t, t^{\prime}\right) \frac{\delta S}{\delta \Psi^{\nu}\left(t^{\prime}\right)}+\frac{\delta}{\delta \Psi^{\nu}\left(t^{\prime}\right)} G^{\mu \nu}\left(t, t^{\prime}\right)\right] d s\right. \\
& \left.+E^{\mu}{ }_{i}\left(t, t^{\prime}\right) d W^{i}\left(t^{\prime}, s\right)\right\}\left.\right|_{\Psi(\cdot)=\Psi(\cdot, s)} .
\end{aligned}
$$

which are distinguished from (3.7) with respect to a different form of the Ito term. We remark that $G^{\mu \nu}$ is assumed to be positive as above with the determinant $G$ being arbitrary. It follows from (3.12) that $G^{\mu \nu}$ factorizes in the corresponding Fokker-Planck operator

$$
L[\Psi]=\int_{\mathbf{R}^{2}} d t d t^{\prime} \frac{\delta}{\delta \Psi^{\mu}(t)} G^{\mu \nu}\left(t, t^{\prime}\right)\left[\frac{\delta S}{\delta \Psi^{\nu}\left(t^{\prime}\right)}+\frac{\delta}{\delta \Psi^{\nu}\left(t^{\prime}\right)}\right]
$$

so that the equilibrium distribution is given independently of the explicit form of $G^{\mu \nu}$ by

$$
\rho[\Psi]_{\text {equil. }}=\frac{e^{-S}}{\int D \Psi e^{-S}} .
$$




\section{The geometrical structure of the helix model}

The helix model is defined by the Lagrange density

$L(t)=\frac{1}{2}\left[\left(\dot{\varphi}^{1}(t)-A(t) \varphi^{2}(t)\right)^{2}+\left(\dot{\varphi}^{2}(t)+A(t) \varphi^{1}(t)\right)^{2}+\left(\dot{\varphi}^{3}(t)-A(t)\right)^{2}\right]-\frac{1}{2}\left[\left(\varphi^{1}(t)\right)^{2}+\left(\varphi^{2}(t)\right)^{2}\right]$

where the dot denotes time derivation and the fields

$$
\vec{\varphi}(t)=\left(\underline{\varphi}(t), \varphi^{3}(t)\right)=\left(\varphi^{1}(t), \varphi^{2}(t), \varphi^{3}(t)\right)
$$

and $A(t)$ are regarded as elements of the function spaces $\mathcal{E}=C^{\infty}\left(\mathbf{R}, \mathbf{R}^{3}\right)$ and $\mathcal{A}=$ $C^{\infty}(\mathbf{R}, \mathbf{R})$, respectively.

Let $\mathcal{G}=C^{\infty}(\mathbf{R}, \mathbf{R})$ denote the abelian group of local gauge transformations where $\left(g_{1} \cdot g_{2}\right)(t)=g_{1}(t)+g_{2}(t)$ gives the group structure. Consider the following transformation $\mathcal{R}$ of $\mathcal{G}$ on the configuration space $\mathcal{E} \times \mathcal{A}$

$$
\mathcal{R}(\vec{\varphi}, A, g)(t)=\left(R(g(t)) \underline{\varphi}(t), \varphi^{3}(t)-g(t), A(t)-\dot{g}(t)\right)
$$

where $g \in \mathcal{G}$ and

$$
R(g(t))=\left(\begin{array}{rr}
\cos g(t) & -\sin g(t) \\
\sin g(t) & \cos g(t)
\end{array}\right) .
$$

The Lagrange density (4.1) is easily verified to be invariant under these transformations and therefore it descends to a function on the quotient space $\mathcal{E} \times \mathcal{G} \mathcal{A}:=(\mathcal{E} \times \mathcal{A}) / \mathcal{G}$ which is obtained by factoring out the gauge degrees of freedom. The generator of infinitesimal gauge transformations is given by the vector field $Z_{\xi}(\Phi)$ with components $Z^{i}\left(t^{\prime}, t\right)$

$$
\begin{aligned}
Z_{\xi}(\Phi) & =\int_{\mathbf{R}} d t\left[-\xi(t) \varphi^{2}(t) \frac{\delta}{\delta \varphi^{1}(t)}+\xi(t) \varphi^{1}(t) \frac{\delta}{\delta \varphi^{2}(t)}-\xi(t) \frac{\delta}{\delta \varphi^{3}(t)}-\dot{\xi}(t) \frac{\delta}{\delta A(t)}\right] \\
& =\int_{\mathbf{R}^{2}} d t d t^{\prime} \xi\left(t^{\prime}\right) Z^{i}\left(t^{\prime}, t\right) \frac{\delta}{\delta \Phi^{i}(t)}
\end{aligned}
$$

on $\mathcal{E} \times \mathcal{A}$, where $\xi \in$ Lie $\mathcal{G}=C^{\infty}(\mathbf{R}, \mathbf{R})$ is an arbitrary element of the Lie algebra of the gauge group and where we defined

$$
\Phi(t)=(\vec{\varphi}(t), A(t))=\left(\varphi^{1}(t), \varphi^{2}(t), \varphi^{3}(t), A(t)\right)
$$


The transformation properties (4.2) suggest to view the fields $A(t)$ and $\varphi(t)$ as gauge field and matter fields, respectively, so that the helix model can be interpreted as an example for a $0+1$ dimensional gauge theory on the real line $\mathbf{R}$ coupled with three matter fields. From a more mathematical point of view, the space $\mathcal{A}$ can be identified with the affine space of connections on the trivial principal fiber bundle over the base $\mathbf{R}$ with the additive group of real numbers as corresponding structure group and the matter fields can then be realized as sections of a particular vector bundle with fiber $\mathbf{R}^{3}$ associated to that given principal bundle.

The true configuration space $\mathcal{E} \times_{\mathcal{G}} \mathcal{A}$ is a smooth manifold which follows from the fact that the gauge group $\mathcal{G}$ acts freely on $\mathcal{E} \times \mathcal{A}$, which means that the equation $\mathcal{R}(\vec{\varphi}, A, g)=$ $(\vec{\varphi}, A)$ for arbitrary fields $(\varphi, A) \in \mathcal{E} \times \mathcal{A}$ only admits the trivial solution $g=0$. Moreover the projection $\pi: \mathcal{E} \times \mathcal{A} \rightarrow \mathcal{E} \times \mathcal{G} \mathcal{A}$, with

$$
\pi(\vec{\varphi}, A)=[\vec{\varphi}, A]
$$

where $[\vec{\varphi}, A]$ denotes the equivalence class of $(\vec{\varphi}, A)$ with respect to the action $\mathcal{R}$, provides the structure of a principal fiber bundle with total space $\mathcal{E} \times \mathcal{A}$, base manifold $\mathcal{E} \times \mathcal{G} \mathcal{A}$ and structure group $\mathcal{G}$.

In this paper we aim at a nonperturbative application of the stochastic quantization scheme to the helix model. In order to proceed it will turn out to be of great importance to rewrite the stochastic process in terms of gauge invariant and gauge dependent fields. However, from a mathematical viewpoint, such a rewriting really can be performed if and only if the principal fiber bundle $\pi: \mathcal{E} \times \mathcal{A} \rightarrow \mathcal{E} \times_{\mathcal{G}} \mathcal{A}$ is trivializable. In this case every field configuration $(\vec{\varphi}, A)$ can be represented in terms of coordinates parametrizing $\mathcal{E} \times_{\mathcal{G}} \mathcal{A}$ and $\mathcal{G}$, respectively.

In order to prove the triviality of the bundle we necessarily have to find a global section, that means a map $\sigma: \mathcal{E} \times \mathcal{G} \mathcal{A} \rightarrow \mathcal{E} \times \mathcal{A}$ satisfying $\pi \circ \sigma=i d$. We will study two specific examples for such global sections.

First we can easily verify that $\sigma_{1}: \mathcal{E} \times \mathcal{G} \mathcal{A} \rightarrow \mathcal{E} \times \mathcal{A}$ given by

$$
\sigma_{1}([\vec{\varphi}, A])=\left(R\left(\varphi^{3}\right) \underline{\varphi}, 0, A-\dot{\varphi}^{3}\right)
$$


is indeed a global section, corresponding in physical terms to the axial gauge $\varphi^{3}=0$.

However we shall display another global section of the bundle which implies the temporal gauge $A=0$. For this case let us take an arbitrary but fixed function $h \in C^{\infty}(\mathbf{R}, \mathbf{R})$ with the property that $\int_{\mathbf{R}} d t h(t)=1$ and $\int_{\mathbf{R}} d t h(t)^{2}=1$. The map $\sigma_{2}: \mathcal{E} \times_{\mathcal{G}} \mathcal{A} \rightarrow \mathcal{E} \times \mathcal{A}$ given by

$$
\sigma_{2}([\vec{\varphi}, A])=\left(R(F(\vec{\varphi}, A)) \underline{\varphi}, \varphi^{3}-F(\vec{\varphi}, A), 0\right)
$$

where $F: \mathcal{E} \times \mathcal{A} \rightarrow \mathcal{G}$ is defined by

$$
F(\vec{\varphi}, A)(t):=\int_{\mathbf{R}} d t^{\prime} h\left(t^{\prime}\right)\left[\int_{t^{\prime}}^{t} d t^{\prime \prime} A\left(t^{\prime \prime}\right)+\varphi^{3}\left(t^{\prime}\right)\right]
$$

yields a global section of $\pi: \mathcal{E} \times \mathcal{A} \rightarrow \mathcal{E} \times \mathcal{G} \mathcal{A}$. To see this, we first note that $\sigma_{2}$ is well defined which follows from $F(R(\vec{\varphi}, A, g))=F(\vec{\varphi}, A)-g$. To prove finally that $\sigma_{2}$ is a section we have to use the fact that $\dot{F}(\vec{\varphi}, A)=A$.

For a given section $\sigma_{i}$ we have a diffeomorphism $\chi_{i}:\left(\mathcal{E} \times_{\mathcal{G}} \mathcal{A}\right) \times \mathcal{G} \rightarrow \mathcal{E} \times \mathcal{A}$ given by

$$
\chi_{i}([\vec{\varphi}, A], g)=\mathcal{R}\left(\sigma_{i}([\vec{\varphi}, A]), g\right)
$$

where $i=1,2$. Let $\omega_{\left(\sigma_{i}\right)}: \mathcal{E} \times \mathcal{A} \rightarrow \mathcal{G}$ be the function which is uniquely determined by the equation

$$
(\vec{\varphi}, A)=\mathcal{R}\left(\sigma_{i}(\pi(\vec{\varphi}, A)), \omega_{\left(\sigma_{i}\right)}(\vec{\varphi}, A)\right) .
$$

Explicitely we find

$$
\omega_{\left(\sigma_{i}\right)}(\vec{\varphi}, A)=\sigma_{i}^{3}([\vec{\varphi}, A])-\varphi^{3},
$$

where $\sigma_{i}^{3}([\vec{\varphi}, A])$ denotes the third component of the i-th section $\sigma_{i}$. For the two choices (4.5) and (4.6) for the sections $\sigma_{i}$ we have

$$
\omega_{\left(\sigma_{1}\right)}(\vec{\varphi}, A)=-\varphi^{3}
$$

and

$$
\omega_{\left(\sigma_{2}\right)}(\vec{\varphi}, A)=-F(\vec{\varphi}, A),
$$

respectively. We remark that under a gauge transformation $\omega_{\left(\sigma_{i}\right)}$ transforms simply like

$$
\omega_{\left(\sigma_{i}\right)}(\mathcal{R}(\vec{\varphi}, A, g))=\omega_{\left(\sigma_{i}\right)}(\vec{\varphi}, A)+g
$$


and for later use also mention the important property

$$
\omega_{\left(\sigma_{i}\right)}\left(\sigma_{i}([\vec{\varphi}, A])\right)=0 .
$$

Now it can be verified straightforwardly that the inverse mapping $\chi_{i}^{-1}: \mathcal{E} \times \mathcal{A} \rightarrow$ $(\mathcal{E} \times \mathcal{G} \mathcal{A}) \times \mathcal{G}$ is given by

$$
\chi_{(i)}^{-1}(\vec{\varphi}, A)=\left([\vec{\varphi}, A], \omega_{\left(\sigma_{i}\right)}(\vec{\varphi}, A)\right)
$$

and it is therefore globally possible to rewrite every field configuration in the appropriate manner. In physical terms the triviality of the principal fiber bundle $\pi: \mathcal{E} \times \mathcal{A} \rightarrow \mathcal{E} \times \mathcal{G} \mathcal{A}$ means that the helix model does not suffer from a topological Gribov problem.

However, for further use it is inconvenient to rewrite all fields in terms of equivalence classes on $\mathcal{E} \times \mathcal{G} \mathcal{A}$. Therefore it seems to be more appropriate to introduce coordinates which parametrize a space diffeomorphic to the orbit space $\mathcal{E} \times_{\mathcal{G}} \mathcal{A}$ which also may be embedded into the original configuration space $\mathcal{E} \times \mathcal{A}$. A natural choice is provided by the image of the global section $\sigma$.

Let $\Sigma_{\left(\sigma_{i}\right)}=\operatorname{im} \sigma_{i}$ denote the gauge fixing surface in $\mathcal{E} \times \mathcal{A}$ for a given section $\sigma_{i}$ then we can consider the isomorphic principal fiber bundle $\pi_{\left(\sigma_{i}\right)}: \mathcal{E} \times \mathcal{A} \rightarrow \Sigma_{\left(\sigma_{i}\right)}$ with projection $\pi_{\left(\sigma_{i}\right)}=\sigma_{i} \circ \pi$,

$$
\pi_{\left(\sigma_{i}\right)}(\vec{\varphi}, A)=\sigma_{i}([\vec{\varphi}, A]),
$$

total space $\mathcal{E} \times \mathcal{A}$, and base manifold $\Sigma_{\left(\sigma_{i}\right)}$, which can be treated as a submanifold in $\mathcal{E} \times \mathcal{A}$ (see Fig. 1 for these geometrical structures).

At this point we want to remark that due to (4.14) the fields $(\vec{\varphi}, A)$ on the gauge fixing surface $\Sigma_{\left(\sigma_{i}\right)}$ can equivalently be characterized by the implicit condition

$$
\omega_{\left(\sigma_{i}\right)}(\vec{\varphi}, A)=0 .
$$

For our first choice (4.5) of the global section $\sigma_{1}$ we simply obtain from (4.11), (4.17) that $\varphi^{3}=0$, whereas for $\sigma_{2}$ defined in (4.6) we derive

$$
A=0 \quad \text { as well as } \quad \int_{\mathbf{R}} d t h(t) \varphi^{3}(t)=0
$$


for $h$ with the properties as given above.

Corresponding to the bundle $\pi_{\left(\sigma_{i}\right)}: \mathcal{E} \times \mathcal{A} \rightarrow \Sigma_{\left(\sigma_{i}\right)}$ a section can be chosen to be the identity mapping so that in analogy to (4.8) the bundle trivialization $\chi_{\left(\sigma_{i}\right)}: \Sigma_{\left(\sigma_{i}\right)} \times \mathcal{G} \rightarrow$ $\mathcal{E} \times \mathcal{A}$ is given by

$$
\chi_{\left(\sigma_{i}\right)}\left(\sigma_{i}([\vec{\varphi}, A]), g\right)=\mathcal{R}\left(\sigma_{i}([\vec{\varphi}, A]), g\right) .
$$

Corresponding to (4.10) and (4.15) the inverse mapping $\chi_{\left(\sigma_{i}\right)}^{-1}: \mathcal{E} \times \mathcal{A} \rightarrow \Sigma_{\left(\sigma_{i}\right)} \times \mathcal{G}$ reads

$$
\chi_{\left(\sigma_{i}\right)}^{-1}(\vec{\varphi}, A)=\left(\sigma_{i}([\vec{\varphi}, A]), \omega_{\left(\sigma_{i}\right)}(\vec{\varphi}, A)\right) .
$$

In subsequent chapters we will use exactly this diffeomorphism $\mathcal{E} \times \mathcal{A} \cong \Sigma_{\left(\sigma_{i}\right)} \times \mathcal{G}$ for studying the stochastic process associated with the helix model. This result provides the way to separate the original process in the configuration space $\mathcal{E} \times \mathcal{A}$ into two subprocesses, one of which is formulated just in terms of fields $(\vec{\varphi}, A) \in \Sigma_{\left(\sigma_{i}\right)}$ whereas the other process is involving gauge dependent fields as well. Since this diffeomorphism explicitly depends on the chosen section $\sigma_{(i)}$, the corresponding processes will be related with the actual gauge fixing.

Let us introduce general coordinates $\vec{\Psi}_{\left(\sigma_{i}\right)}$ on $\Sigma_{\left(\sigma_{i}\right)}$

$$
\vec{\Psi}_{\left(\sigma_{i}\right)}=\left(\underline{\Psi}_{\left(\sigma_{i}\right)}, \Psi_{\left(\sigma_{i}\right)}^{3}\right)=\left(\Psi_{\left(\sigma_{i}\right)}^{1}, \Psi_{\left(\sigma_{i}\right)}^{2}, \Psi_{\left(\sigma_{i}\right)}^{3}\right)
$$

and a coordinate $\Psi_{\left(\sigma_{i}\right)}^{4}$ parametrizing $\mathcal{G}$, which we combine into

$$
\Psi_{\left(\sigma_{i}\right)}=\left(\vec{\Psi}_{\left(\sigma_{i}\right)}, \Psi_{\left(\sigma_{i}\right)}^{4}\right)=\left(\Psi_{\left(\sigma_{i}\right)}^{1}, \Psi_{\left(\sigma_{i}\right)}^{2}, \Psi_{\left(\sigma_{i}\right)}^{3}, \Psi_{\left(\sigma_{i}\right)}^{4}\right) .
$$

From (4.20) we trivially deduce 3-dimensional coordinates on $\Sigma_{\left(\sigma_{i}\right)}$ by observing that for each of the two choices (4.5), (4.6) the 4 -vector $\sigma_{i}([\vec{\varphi}, A])$ has one vanishing component; $\Psi_{\left(\sigma_{i}\right)}^{4}$ is taken to be $\omega_{\left(\sigma_{i}\right)}(\vec{\varphi}, A)$.

For the particular section $\sigma_{1}$ in (4.5), (4.20) yields

$$
\begin{aligned}
& \underline{\Psi}_{\left(\sigma_{1}\right)}=R\left(\varphi^{3}\right) \underline{\varphi} \\
& \Psi_{\left(\sigma_{1}\right)}^{3}=A-\dot{\varphi}^{3} \\
& \Psi_{\left(\sigma_{1}\right)}^{4}=\omega_{\left(\sigma_{1}\right)}(\vec{\varphi}, A)=-\varphi^{3}
\end{aligned}
$$


with inverse

$$
\begin{aligned}
\underline{\varphi} & =R\left(\Psi_{\left(\sigma_{1}\right)}^{4}\right) \underline{\Psi}_{\left(\sigma_{1}\right)} \\
\varphi^{3} & =-\Psi_{\left(\sigma_{1}\right)}^{4} \\
A & =\Psi_{\left(\sigma_{1}\right)}^{3}-\dot{\Psi}_{\left(\sigma_{1}\right)}^{4} .
\end{aligned}
$$

For $\sigma_{2}$ as defined in (4.6) the corresponding coordinate transformation (4.20) reads

$$
\begin{aligned}
& \underline{\Psi}_{\left(\sigma_{2}\right)}=R(F(\vec{\varphi}, A)) \underline{\varphi} \\
& \Psi_{\left(\sigma_{2}\right)}^{3}=\varphi^{3}-F(\vec{\varphi}, A) \\
& \Psi_{\left(\sigma_{2}\right)}^{4}=-F(\vec{\varphi}, A)
\end{aligned}
$$

with inverse

$$
\begin{aligned}
\underline{\varphi} & =R\left(\Psi_{\left(\sigma_{2}\right)}^{4}\right) \underline{\Psi}_{\left(\sigma_{2}\right)} \\
\varphi^{3} & =\widetilde{\Psi}_{\left(\sigma_{2}\right)}^{3}-\Psi_{\left(\sigma_{2}\right)}^{4} \\
A & =-\dot{\Psi}_{\left(\sigma_{2}\right)}^{4}
\end{aligned}
$$

where

$$
\widetilde{\Psi}_{\left(\sigma_{2}\right)}^{3}(t)=\int_{\mathbf{R}} d t^{\prime}\left[\delta\left(t-t^{\prime}\right)-h(t) h\left(t^{\prime}\right)\right] \Psi_{\left(\sigma_{2}\right)}^{3}\left(t^{\prime}\right)
$$

Notice that corresponding to (4.17) and (4.18) the coordinates $\vec{\Psi}_{\left(\sigma_{2}\right)}$ on $\Sigma_{\left(\sigma_{2}\right)}$ fulfill $\int_{\mathbf{R}} d t h(t) \Psi_{\left(\sigma_{2}\right)}^{3}(t)=0$ which explains the presence of the projection operator $\delta\left(t-t^{\prime}\right)-$ $h(t) h\left(t^{\prime}\right)$ acting on $\Psi_{\left(\sigma_{2}\right)}^{3}$ in (4.24) and (4.25). This operator is not needed in (4.23) as $\Psi_{\left(\sigma_{2}\right)}^{3}$ defined in terms of $(\vec{\varphi}, A)$ automatically fulfills the required relation .

In the new coordinates, gauge transformations are given purely as translations, i.e. $\left(\vec{\Psi}_{\left(\sigma_{i}\right)}, \Psi_{\left(\sigma_{i}\right)}^{4}\right) \rightarrow\left(\vec{\Psi}_{\left(\sigma_{i}\right)}, \Psi_{\left(\sigma_{i}\right)}^{4}+g\right)$ where $g \in \mathcal{G}$. Correspondingly, the generator for an infinitesimal gauge transformation $\xi \in \operatorname{Lie} \mathcal{G}$

$$
Z_{\xi}\left(\Psi_{\left(\sigma_{i}\right)}\right)=\int_{\mathbf{R}} d t \xi(t) \frac{\delta}{\delta \Psi_{\left(\sigma_{i}\right)}^{4}(t)}
$$

is just the invariant vector field along the gauge group. 
Let us discuss our findings of this chapter in more detail: We have seen that the principal fiber bundle associated with the helix model is trivializable and so does not suffer from a Gribov problem. To clarify this point we recall that in strict mathematical terms (as analyzed by Singer [9] in the case of Yang-Mills theory) one speaks about a Gribov problem if the projection from the original configuration space onto the space of gauge orbits gives a non-trivializable principal fiber bundle. In this case it is not possible to find a separation of any field configuration in terms of gauge invariant and gauge variant fields in a global way. Such a separation can be done only locally and as a result there may occur ambiguities in the choice of appropriate coordinates on the local patches of the orbit space which have nonvoid intersection.

Our main idea was to select a gauge fixing surface in the original configuration space. This choice was naturally given by taking a particular section in the corresponding principal fiber bundle. The gauge fixing surface was then characterized as a set of solution of a certain function (4.11) on the configuration space depending, in fact, on the chosen section.

We recall at this place that recently several papers have been published [13, 14] which take the helix model as an (explicitly soluble) example for analyzing the Gribov problem. The apparent discrepancy to our result can be traced back to different concepts of definitions: There exists of course the possibility to define an arbitrary function on the configuration space, consider the set of points where it vanishes and ask if this set is a diffeomorphic copy of the gauge orbit space. It is precisely this route of analysis which has been followed in the above mentioned papers. Depending on the actual form of the given function, it may be possible to find at least a certain subset of solutions which is diffeomorphic to a subset of the orbit space. In that case the theory was said to possess a Gribov ambiguity. We prefer to interpret the kind of Gribov ambiguity which is talked about in this context as being of an artificial kind.

In a following paper [32] we shall present a stochastic quantization analysis of this artificial Gribov problem associated with sections of the bundle $\pi: \mathcal{E} \times \mathcal{A} \rightarrow \mathcal{E} \times \mathcal{G} \mathcal{A}$ which can be defined only locally. 


\section{$5 \quad$ Generalized stochastic gauge fixing}

In this section we review the stochastic quantization procedure of gauge (field) theories and present our generalisation of the stochastic gauge fixing procedure of Zwanziger [17.

In order to work out the new features of our modified approach we review at the beginning the Parisi-Wu stochastic quantization scheme [1] for gauge theories and Zwanziger's original gauge fixing procedure. This summary serves as a complement to our general introductory remarks in sect. 1) and to our short review in sect. 3) of the stochastic quantization of non-gauge models.

We collectively denote by $\Phi^{k}$ the pure gauge, as well as matter fields of the given gauge model. For reasons of simplicity we choose a notation in correspondance with the helix model; in specific we introduce 4 fields depending on the (Euclidean) time coordinate $t$, as the helix model is defined in terms of 4 fields in $0+1$ dimensions. We remark that a generalization to more space time dimension and to more general gauge models is immediate. According to the setting of stochastic quantization within the Langevin picture the fields are defined to depend additionally on the stochastic time coordinate $s$ as well; explicitly this amounts to substitute $\Phi^{k}(t)$ by $\Phi^{k}(s, t)$.

The crucial point of the Parisi-Wu approach for the gauge theory case is to demand that the stochastic time evolution of the fields is given again by a Langevin equation of the form

$$
d \Phi^{i}(t, s)=-\left.\delta^{i j} \frac{\delta S}{\delta \Phi^{j}(t)}\right|_{\Phi(t)=\Phi(t, s)} d s+d W^{i}
$$

where $S$ denotes the original (Euclidean) action of the gauge model; it is the unmodified bare action without gauge symmetry breaking terms and without accompanying ghost field terms; in our case of the helix model it is given by the time integral of the Wick rotated form of the Lagrangian (4.1).

We have alread remarked in the introductiory section that in the case of a gauge theory the stochastic process defined by (5.1) undergoes undamped diffusion and does not approach an equilibrium distribution. Related to this fact is that a Fokker-Planck

formulation for the $\Phi^{k}$ is not possible because the gauge invariance of the action leads to 
divergencies in the normalization condition of the Fokker-Planck density.

We now discuss Zwanziger's modified formulation [17] of the Parisi-Wu scheme: The stochastic gauge fixing procedure consists in adding an additional drift force to the Langevin equation (5.1) which acts tangentially to the gauge orbits. This additional term generally can be expressed by the gauge generator and an arbitrary function $\alpha$; in the specific case of the helix model the components of the gauge generator are defined by (4.3) so that the modified Langevin equation reads as follows

$$
d \Phi^{i}(t, s)=-\left.\left[\delta^{i j} \frac{\delta S}{\delta \Phi^{j}(t)}+\int_{\mathbf{R}} d t^{\prime} Z^{i}\left(t, t^{\prime}\right) \alpha\left(t^{\prime}\right)\right]\right|_{\Phi(\cdot)=\Phi(\cdot, s)} d s+d W^{i}
$$

Let us note two remarkable consequences of the extra term in (5.2)

a) The expectation values of gauge invariant observables remain unchanged for any choice of the function $\alpha$ (see below for the explicit demonstration contained in the discussion of our generalized stochastic gauge fixing procedure)

b) For specific choices of the - in principle - arbitrary function $\alpha$ the gauge modes' diffusion is damped along the gauge orbits. As a consequence the Fokker-Planck density can be normalized and arbitrary expectation values of the fields are relaxing exponentially in the stochastic time s to equilibrium values. We remind that this situation is in contrast to the Parisi- Wu approach, where for expectation values of gauge variant observables no equilibrium values could be attained.

We present now our generalization of Zwanziger's stochastic gauge fixing procedure by adding a specific drift term which not only has tangential components along the gauge orbits; in addition we modify the Wiener process itself. Due to this generalization we are able to introduce more than just only one extra function $\alpha$, in fact we additionally introduce 4 arbitrary functions $\beta_{k}$. Our generalization is done in such a way that expectation values of gauge invariant observables again remain untouched.

Our generalized Langevin equation reads

$$
d \Phi^{i}(t, s)=-\left[\delta^{i j} \frac{\delta S}{\delta \Phi^{j}(t)}+\int_{\mathbf{R}} d t^{\prime} Z^{i}\left(t, t^{\prime}\right) \alpha\left(t^{\prime}\right)\right.
$$




$$
\begin{aligned}
& \left.+\int_{\mathbf{R}^{2}} d t_{1} d t_{2} \frac{\delta Z^{i}\left(t, t_{1}\right)}{\delta \Phi^{k}\left(t_{2}\right)} \zeta^{k}\left(t_{1}, t_{2}\right)\right]\left.\right|_{\Phi(\cdot)=\Phi(\cdot, s)} d s \\
& +\left.\int_{\mathbf{R}} d t_{2}\left[\delta^{i}{ }_{k} \delta\left(t-t_{2}\right)+\int_{\mathbf{R}} d t_{1} Z^{i}\left(t, t_{1}\right) \beta_{k}\left(t_{1}, t_{2}\right)\right]\right|_{\Phi(\cdot)=\Phi(\cdot, s)} d W^{k}\left(t_{2}, s\right)
\end{aligned}
$$

here we introduced $\zeta^{k}\left(t_{1}, t_{2}\right)$ as a shorthand notation of

$$
\zeta^{k}\left(t_{1}, t_{2}\right)=2 \delta^{k \ell} \beta_{\ell}\left(t_{1}, t_{2}\right)+\int_{\mathbf{R}^{2}} d t_{3} d t_{4} Z^{k}\left(t_{2}, t_{3}\right) \beta_{\ell}\left(t_{3}, t_{4}\right) \delta^{\ell m} \beta_{m}\left(t_{1}, t_{4}\right)
$$

We see that the new drift term clearly is not acting tangential to the gauge orbit; its rather complicated structure is necessary for leaving unchanged gauge invariant expectation values. We prove that this is indeed so.

Suppose that $F$ is a gauge invariant functional of the fields $\Phi^{k}$, so that

$$
\int d t \frac{\delta F}{\delta \Phi^{i}(t)} Z^{i}\left(t, t^{\prime}\right)=0
$$

We consider now the difference of the expectation values of $F$ when we first use the generalized Langevin equation (5.3) and second the original Parisi-Wu equation (5.1), which is just the $\alpha=0, \beta_{k}=0$ contribution of (5.3). With the Ito rule (2.7) we find straightforwardly

$$
\begin{aligned}
& \langle\langle d F\rangle\rangle-\left.\langle\langle d F\rangle\rangle\right|_{\alpha=0, \beta_{k}=0}=\left\langle\left\langle\int _ { \mathbf { R } ^ { 2 } } d t _ { 1 } d t _ { 2 } \left[\frac{\delta F}{\delta \Phi^{i}\left(t_{1}\right)} Z^{i}\left(t_{1}, t_{2}\right) \alpha\left(t_{2}\right)\right.\right.\right. \\
& \left.\left.\left.\quad+\int_{\mathbf{R}} d t_{3} \frac{\delta}{\delta \Phi^{k}\left(t_{3}\right)}\left(\frac{\delta F}{\delta \Phi^{i}\left(t_{1}\right)} Z^{i}\left(t_{1}, t_{2}\right)\right) \zeta^{k}\left(t_{2}, t_{3}\right)\right]\left.\right|_{\Phi(\cdot)=\Phi(\cdot, s)}\right\rangle\right\rangle d s
\end{aligned}
$$

which is zero, as each term vanishes separately due the gauge invariance of $F$.

Our next task is to discuss the issue of stochastic gauge fixing in terms of the FokkerPlanck equation. Zwanziger originally worked in this scheme to derive his stochastic gauge fixing procedure; we will now generalize his formulation.

A remarkable advantage of the Fokker-Planck scheme in comparison to the Langevin formulation is that equilibrium properties of the system may directly be studied in a nonperturbative way. By this we mean that one neither needs to explicitly find the solution of the stochastic differential equation of the fields $\Phi^{k}$, nor is it necessary to solve 
the Fokker-Planck equation for the probability distribution $\rho$ for all stochastic times $s$. What, however, one may directly be able to achieve is to extract from the Fokker-Planck equation just the equilibrium distribution. Surely one must be cautious to check that all conditions for the stochastic process really to relax to this equilibrium distribution are fulfilled.

We will demonstrate below that our generalized stochastic gauge fixing formulation will indeed allow us to extract in a nonperturbative manner an equilibrium Fokker-Planck probability distribution which defines a standard field theory path integral density for the helix model.

First we want to repeat within the Fokker-Planck approach that gauge invariant expectation values remain unchanged when our generalized stochastic gauge fixing procedure is applied. We have to study the stochastic time development of gauge invariant expectation values and therefore are interested in the form of the adjoint Fokker-Planck operator $L^{\dagger}$. Corresponding to the Langevin equation (5.3) and following the general discussion (2.1) and (2.12) we obtain

$$
\begin{aligned}
L^{\dagger}[\Phi]= & \int_{\mathbf{R}} d t\left[-\frac{\delta S}{\delta \Phi^{i}(t)}+\frac{\delta}{\delta \Phi^{i}(t)}\right] \delta^{i j} \frac{\delta}{\delta \Phi^{j}(t)} \\
& +\int_{\mathbf{R}^{2}} d t_{1} d t_{2} Z^{i}\left(t_{1}, t_{2}\right) \alpha\left(t_{2}\right) \frac{\delta}{\delta \Phi^{i}\left(t_{1}\right)} \\
& +\int_{\mathbf{R}^{3}} d t_{1} d t_{2} d t_{3} \zeta^{k}\left(t_{2}, t_{3}\right) \frac{\delta}{\delta \Phi^{k}\left(t_{3}\right)}\left(Z^{i}\left(t_{1}, t_{2}\right) \frac{\delta}{\delta \Phi^{i}\left(t_{1}\right)}\right) .
\end{aligned}
$$

We recognize that each of the last two terms is a differential operator acting along the gauge orbits so that it vanishes when it is applied to gauge invariant observables. Thus we have demonstrated within the Fokker-Planck formulation as well that expectation values of gauge invariant observables are not affected by our modifications.

Comparing with Zwanziger's original stochastic gauge fixing procedure our generalized scheme amounts to adding to the (adjoint) Fokker-Planck operator not only a gauge symmetry preserving part which is linear in the field derivatives, but also a gauge symmetry preserving part which contains quadratic derivatives. In other words not only the drift term but also the diffusion term of the (adjoint) Fokker-Planck operator gets modi- 
fied; the modification of the latter corresponds precisely to the modifcation of the Wiener process of the Langevin equation (5.3).

It is of crucial importance in Zwanziger's approach to draw advantage of the fact that the expectation values of gauge invariant quantities are independent of the new drift term, which in specific means that they are independent of the function $\alpha$. One may chose now such a specific value for it that the functional integrations along the gauge orbits receive proper damping. Equivalently the gauge modes' contributions to expectation values are seen to converge rapidly, i.e. exponentially, in stochastic time. Only with such a proper choice the Fokker-Planck density can be normalized to unity and allows for a true probabilistic interpretation of the stochastic scheme.

We want to discuss the issue of proper damping of the gauge modes within our generalized scheme in the next section.

\section{Damping of the gauge degrees of freedom}

So far we explained our stochastic gauge fixing method in quite general terms, but we now want to consider gauge models where the transition to gauge invariant variables at the one hand and gauge transforming variables on the other hand globally is possible. Geometrically this corresponds to a situation where the configuration space admits a structure of a trivialisable principal fiber bundle. The transition to these new variables is not unique and corresponds to a specific choice of gauge.

In the following we specialize further and discuss the helix model in detail. In this way we can make use of our geometrical analysis in chapter 4) and concentrate on the two sets $(4.15,4.17)$ of field variables $\Psi_{\left(\sigma_{i}\right)}, i=1,2$, which are related to the gauge fixing surfaces $\Phi^{3}=0$ and $A=0$, respectively. We remind that in both cases the gauge dependent variables are changing under gauge transformations just by a simple shift.

We want to investigate how the Langevin equations (5.3) are changed when we transform the original field variables $\Phi$ into one of the sets of new coordinates $\Psi_{\left(\sigma_{i}\right)}$. With respect to these variable changes we introduce the vielbeins $E$ and their inverses $e$, as 
well as the induced inverse metric $G^{\mu \nu}$; see (3.4-3.5), as well as the Appendix for explicit expressions.

With the use of the Ito formula (2.6) the Langevin equations (5.3) are transformed in a similar way as (3.7) into

$$
\begin{aligned}
d \Psi^{\mu}(t, s)= & \int_{\mathbf{R}} d t_{1}\left\{\left[-G^{\mu \nu}\left(t, t_{1}\right) \frac{\delta S}{\delta \Psi^{\nu}\left(t_{1}\right)}+\frac{\delta^{2} \Psi^{\mu}(t)}{\delta \Phi^{i}\left(t_{1}\right) \delta \Phi^{i}\left(t_{1}\right)}\right.\right. \\
& \left.+\int_{\mathbf{R}} d t_{2} E^{\mu}{ }_{i}\left(t, t_{1}\right) Z^{i}\left(t_{1}, t_{2}\right) \alpha\left(t_{2}\right)\right] d s \\
& +\int_{\mathbf{R}} d t_{2} E^{\mu}{ }_{i}\left(t, t_{1}\right)\left[\delta^{i}{ }_{k} \delta\left(t_{1}-t_{2}\right)+\int_{\mathbf{R}} d t_{3} Z^{i}\left(t_{1}, t_{3}\right) \beta_{k}\left(t_{3}, t_{2}\right)\right] d W^{k}\left(t_{2}, s\right) \\
& \left.+\int_{\mathbf{R}^{3}} d t_{2} d t_{3} d t_{4}\left[\frac{\delta}{\delta \Phi^{k}\left(t_{2}\right)}\left(E^{\mu}{ }_{i}\left(t, t_{4}\right) Z^{i}\left(t_{4}, t_{1}\right)\right)\right] \zeta\left(t_{1}, t_{2}\right)\right\}\left.\right|_{\Psi(\cdot)=\Psi(\cdot, s)}
\end{aligned}
$$

Fortunately the last term in this somewhat involved looking expression vanishes. This follows from

$$
Z^{i}\left(t, t^{\prime}\right)=e_{4}^{i}\left(t, t^{\prime}\right)
$$

and the fact that $e$ is the inverse to $E$. The expression (6.2) can be derived by considering that the new coodinates $\Psi$ are gauge invariant except for $\Psi^{4}$ which, however, is transforming only by a linear shift, see the discussion below (4.13). Therefore under an infinitesimal gauge transformation $\xi \in$ Lie $\mathcal{G}$ the fields $\Psi^{\mu}(t)$ transform according to the Lie-derivation along the vector field $Z_{\xi}$,

$$
\begin{aligned}
Z_{\xi}\left(\Psi^{\mu}(t)\right) & =\int_{\mathbf{R}^{2}} d t_{1} d t_{2} \xi\left(t_{1}\right) Z^{i}\left(t_{1}, t_{2}\right) \frac{\delta \Psi^{\mu}(t)}{\delta \Phi^{i}\left(t_{1}\right)} \\
& =\int_{\mathbf{R}^{2}} d t_{1} d t_{2} \xi(t 1) Z^{i}\left(t_{1}, t_{2}\right) E^{\mu}{ }_{i}\left(t, t_{1}\right) \\
& =\delta^{\mu}{ }_{4} \xi(t)
\end{aligned}
$$

so that (6.2) can be obtained. We also have

$$
\int_{\mathbf{R}} d t \frac{\delta E^{\nu}{ }_{i}\left(t, t^{\prime}\right)}{\delta \Psi^{\nu}(t)}=0
$$

which is satisfied by both of the above sets of coordinates $\Psi_{(\sigma)}$ (see the Appendix) so that the Ito term takes the special form

$$
\int_{\mathbf{R}} d t_{1} \frac{\delta^{2} \Psi^{\mu}(t)}{\delta \Phi^{i}\left(t_{1}\right) \delta \Phi^{i}\left(t_{1}\right)}=\int_{\mathbf{R}^{2}} d t_{1} d t_{2} E^{\nu}{ }_{i}\left(t_{2}, t_{1}\right) \frac{\delta E^{\mu}\left(t, t_{1}\right)}{\delta \Psi^{\nu}\left(t_{2}\right)}
$$




$$
=\int_{\mathbf{R}} d t_{2} \frac{\delta}{\delta \Psi^{\nu}\left(t_{2}\right)} G^{\nu \mu}\left(t_{2}, t\right)
$$

Thus we finally find the Langevin equations for the new variables $\Psi_{(\sigma)}$

$$
\begin{aligned}
d \Psi^{\mu}(t, s)= & \int_{\mathbf{R}} d t_{1}\left\{\left[-G^{\mu \nu}\left(t, t_{1}\right) \frac{\delta S}{\delta \Psi^{\nu}\left(t_{1}\right)}+\frac{\delta G^{\nu \mu}\left(t_{1}, t\right)}{\delta \Psi^{\nu}\left(t_{1}\right)}+\delta^{\mu}{ }_{4} \delta\left(t-t_{1}\right) \alpha\left(t_{1}\right)\right] d s\right. \\
& \left.+\left[E^{\mu}{ }_{i}\left(t, t_{1}\right)+\delta^{\mu}{ }_{4} \beta_{i}\left(t, t_{1}\right)\right] d W^{i}\left(t_{1}, s\right)\right\}\left.\right|_{\Psi(\cdot)=\Psi(\cdot, s)} .
\end{aligned}
$$

We recognize that the terms due to the generalized gauge fixing procedure - the terms containing the arbitrary functions $\alpha$ and $\beta_{k}$ - are not affecting the Langevin equations of the gauge independent fields $\Psi_{(\sigma)}^{\bar{\mu}}, \bar{\mu}=1,2,3$

$$
\begin{aligned}
d \Psi^{\bar{\mu}}(t, s)= & \int_{\mathbf{R}} d t_{1}\left\{\left[-G^{\bar{\mu} \bar{\nu}}\left(t, t_{1}\right) \frac{\delta S}{\delta \Psi^{\bar{\nu}}\left(t_{1}\right)}+\frac{\delta G^{\bar{\nu} \bar{\mu}}\left(t_{1}, t\right)}{\delta \Psi^{\bar{\nu}}\left(t_{1}\right)}\right] d s\right. \\
& \left.+E^{\bar{\mu}}{ }_{i}\left(t, t_{1}\right) d W^{i}\left(t_{1}, s\right)\right\}\left.\right|_{\Psi(\cdot)=\Psi(\cdot, s)} .
\end{aligned}
$$

It is important to remark that these Langevin equations are closed among themselves and can be solved independently of the gauge dependent variable $\Psi^{4}$. This may look surprising, as the vielbein $\mathrm{E}$ generally is a gauge dependent matrix $\left(G^{\mu \nu}\right.$, however, is gauge independent by direct inspection). The claimed gauge independence of the above Langevin equations follows because

i) the vielbein $E$ can be split into the product of a gauge invariant matrix and a pure rotation matrix which contains all the gauge dependence (see (A.3) and (A.7))

ii) the Ito-Langevin equations are invariant under rotations of the vielbeins (see [36] and references therein), so that the gauge dependent part of $E$ can just be rotated away.

iii) The gauge independence of $G^{\mu \nu}$ follows generally from (3.5) as a direct consequence of the product form of $E$.

At this point it seems appropriate to notice that equation (6.7) can also be obtained within the pure Parisi-Wu formulation of gauge theories, i.e. without introducing any stochastic gauge fixing terms. In this case the original Langevin equation (5.1) constitutes 
the starting point for introducing the new coordinates $\Psi$, all the steps concerning the application of the Ito formula are the same as above. The formulation (6.7) in terms of only gauge invariant fields is precisely in the spirit of the original paper of Parisi-Wu [1] where the transition to gauge invariant variables and the discarding of the gauge variant variables was suggested. In our case we are, however, particulary interested in connecting the stochastic formalism to the path integral scheme. We therefore prefer to consider the stochastic time evolution of all the variables of the gauge model and continue within the generalized stochastic gauge fixing approach.

In order to derive the Langevin equation of the gauge dependent field $\Psi^{4}$ we use

$$
\int d t^{\prime} \frac{\delta G^{\nu 4}\left(t^{\prime}, t\right)}{\delta \Psi^{\nu}\left(t^{\prime}\right)}=\int d t^{\prime} \frac{\delta E^{4}{ }_{i}\left(t, t^{\prime}\right)}{\delta \Phi^{i}\left(t^{\prime}\right)}=0
$$

This follows from (6.4) and the fact that $\Psi^{4}$ is linear in $\Phi$, see (4.15) and (4.17), as well as (4.6). We obtain

$$
\begin{aligned}
d \Psi^{4}(t, s)= & \int_{\mathbf{R}} d t_{1}\left\{\left[-G^{4 \nu}\left(t, t_{1}\right) \frac{\delta S}{\delta \Psi^{\nu}\left(t_{1}\right)}+\alpha\left(t_{1}\right) \delta\left(t-t_{1}\right)\right] d s\right. \\
& \left.+\left[E^{4}{ }_{k}\left(t, t_{1}\right)+\beta_{k}\left(t, t_{1}\right)\right] d W^{k}\left(t_{1}, s\right)\right\}\left.\right|_{\Psi(\cdot)=\Psi(\cdot, s)} .
\end{aligned}
$$

We note that on the right hand side of (6.9) no gauge dependent terms are present apart of the possibility that the so far arbitrary functions $\alpha$ and $\beta_{k}$ are changing under gauge transformations. Let us suppose, however, for a moment that $\alpha$ is gauge invariant. In this case the drift term of (6.9) is gauge invariant as well and remains unchanged for a stochastic evolution along the gauge orbits, i.e. along the gauge transforming variable $\Psi^{4}$ keeping the other variables fixed. For this stochastic process the drift term of the Langevin equation of $\Psi^{4}$ appears like a constant; it gives rise to a diffusion behaviour and will never allow relaxation to an equilibrium distribution.

But we can convert this diffusion into a well damped stochastic process by conveniently choosing a gauge dependent $\alpha$ : We recall that due to the general philosophy of stochastic gauge fixing, which we have formulated in the previous section, every choice of $\alpha$ (as well as of $\beta_{k}$ ) is permissible.

Out of the possible choices the simplest Langevin equation is obtained if $\alpha$ subtracts off the above gauge invariant drift term and adds a term proportional to $\Psi^{4}$ itself, the 
proportionality factor being built from a gauge invariant and strictly positive integral kernel $\gamma$

$$
\alpha(t)=\int_{\mathbf{R}} d t^{\prime}\left[G^{4 \nu}\left(t, t^{\prime}\right) \frac{\delta S}{\delta \Psi^{\nu}\left(t^{\prime}\right)}-\gamma\left(t, t^{\prime}\right) \Psi^{4}\left(t^{\prime}\right)\right] .
$$

We can suggestively rewrite this damping term by introducing the total action $S_{\text {tot }}$

$$
S_{\mathrm{tot}}=S+\frac{1}{2} \int_{\mathbf{R}} d t\left(\Psi^{4}(t)\right)^{2}
$$

so that the $\Psi^{4}$ Langevin equation becomes

$$
\begin{aligned}
d \Psi^{4}(t, s)= & \int_{\mathbf{R}} d t_{1}\left\{-\gamma\left(t, t_{1}\right) \frac{\delta S_{\text {tot }}}{\delta \Psi^{4}\left(t_{1}\right)}\right. \\
& \left.+\left[E^{4}{ }_{i}\left(t, t_{1}\right)+\beta_{i}\left(t, t_{1}\right)\right] d W^{i}\left(t_{1}, s\right)\right\}\left.\right|_{\Psi(\cdot)=\Psi(\cdot, s)}
\end{aligned}
$$

Let us define a matrix $\widetilde{G}$ by

$$
\begin{aligned}
\widetilde{G}^{\bar{\mu} \bar{\nu}}\left(t_{1}, t_{2}\right) & =G^{\bar{\mu} \bar{\nu}}\left(t_{1}, t_{2}\right) \quad \bar{\mu}, \bar{\nu}=1,2,3 \\
\widetilde{G}^{\bar{\mu} 4}\left(t_{1}, t_{2}\right) & =\widetilde{G}^{4 \bar{\mu}}\left(t_{1}, t_{2}\right)=0 \\
\widetilde{G}^{44}\left(t_{1}, t_{2}\right) & =\gamma\left(t_{1}, t_{2}\right) .
\end{aligned}
$$

Notice that at this moment it is not obvious that $\widetilde{G}$ as defined above is a positive matrix and therefore satisfies the properties of a metric; we will prove it, however, in the next section for a special choice of $\beta_{k}$ and $\gamma$. Since from (6.8)

$$
\frac{\delta \widetilde{G}^{\mu \nu}\left(t_{1}, t_{2}\right)}{\delta \Psi^{\nu}\left(t_{2}\right)}=\frac{\delta G^{\mu \nu}\left(t_{1}, t_{2}\right)}{\delta \Psi^{\nu}\left(t_{2}\right)}
$$

the Langevin equations (6.6) can be rewritten in terms of the total action $S_{\text {tot }}$ and the new metric $\widetilde{G}$ as follows

$$
\begin{aligned}
d \Psi^{\mu}(t, s)= & \int_{\mathbf{R}} d t_{1}\left\{\left[-\widetilde{G}^{\mu \nu}\left(t, t_{1}\right) \frac{\delta S_{\text {tot }}}{\delta \Psi^{\nu}\left(t_{1}\right)}+\frac{\delta \widetilde{G}^{\nu \mu}\left(t_{1}, t\right)}{\delta \Psi^{\nu}\left(t_{1}\right)}\right] d s\right. \\
& \left.+\left[E^{\mu}{ }_{k}\left(t, t_{1}\right)+\delta^{\mu}{ }_{4} \beta_{k}\left(t, t_{1}\right)\right] d W^{k}\left(t_{1}, s\right)\right\}\left.\right|_{\Psi(\cdot)=\Psi(\cdot, s)}
\end{aligned}
$$

Before closing this section we rewrite the adjoint Fokker-Planck operator $L^{\dagger}$ in the new coordinates $\Psi_{(\sigma)}$. From (6.6) we get easily

$$
L^{\dagger}[\Psi]=\int_{\mathbf{R}} d t\left\{\int_{\mathbf{R}} d t_{1}\left[-\frac{\delta S}{\delta \Psi^{\nu}\left(t_{1}\right)}+\frac{\delta}{\delta \Psi^{\nu}\left(t_{1}\right)}\right] G^{\mu \nu}\left(t_{1}, t\right) \frac{\delta}{\delta \Psi^{\mu}(t)}+\alpha(t) \frac{\delta}{\delta \Psi^{4}(t)}\right.
$$




$$
\left.+\int_{\mathbf{R}^{2}} d t_{1} d t_{2} \beta_{k} \delta^{k \ell}\left[2 E_{\ell}^{\nu}\left(t_{1}, t_{2}\right) \frac{\delta^{2}}{\delta \Psi^{\nu}\left(t_{1}\right) \delta \Psi^{4}(t)}+\beta_{\ell}\left(t_{1}, t_{2}\right) \frac{\delta^{2}}{\delta \Psi^{4}\left(t_{1}\right) \delta \Psi^{4}(t)}\right]\right\} .
$$

It is evident that the additional terms due to our modifications exhaust the most general structure of the adjoint Fokker-Planck operator compatible with gauge invariance.

We finally rewrite $L^{\dagger}$ upon inserting the special value (6.10) of $\alpha$ as

$$
\begin{aligned}
L^{\dagger}[\Psi]= & \int_{\mathbf{R}^{2}} d t d t_{1}\left\{\left[-\frac{\delta S_{\text {tot }}}{\delta \Psi^{\nu}\left(t_{1}\right)}+\frac{\delta}{\delta \Psi^{\nu}\left(t_{1}\right)}\right] \widetilde{G}^{\nu \mu}\left(t_{1}, t\right) \frac{\delta}{\delta \Psi^{\mu}(t)}\right. \\
& +\left[G^{\mu \nu}\left(t, t_{1}\right)-\widetilde{G}^{\mu \nu}\left(t, t_{1}\right)\right] \frac{\delta^{2}}{\delta \Psi^{\nu}\left(t_{1}\right) \delta \Psi^{\mu}(t)} \\
& \left.+\int_{\mathbf{R}} d t_{2} \beta_{k} \delta^{k \ell}\left[2 E_{\ell}^{\nu}\left(t_{1}, t_{2}\right) \frac{\delta^{2}}{\delta \Psi^{\nu}\left(t_{1}\right) \delta \Psi^{4}(t)}+\beta_{\ell}\left(t_{1}, t_{2}\right) \frac{\delta^{2}}{\delta \Psi^{4}\left(t_{1}\right) \delta \Psi^{4}(t)}\right]\right\} .
\end{aligned}
$$

In retrospect the use of the new coordinates $\Psi_{(\sigma)}$ has turned out to be successful in order to analyze the damping behaviour of the stochastic process. It is explicitly shown that the process along the gauge group, although initially giving rise to pure diffusion, can be damped by an appropriate tuning of the gauge fixing parameter $\alpha$.

\section{The equilibrium distribution}

In this section we exploit the remaining freedom for the choice of the positive kernel $\gamma$ and the vector $\beta_{k}$ to define a specific stochastic process of which we are able to derive the equilibrium distribution.

We repeat once more that the legitimation for the freedom of choosing $\gamma$ and $\beta_{k}$ was given in section 5 where we proved that the expectation values of gauge invariant observables are independent of $\gamma$ and $\beta_{k}$. Therefore, if we are able to find just one stochastic process - defined in terms of specific functions $\alpha$ and $\beta_{k}$ - where the equilibrium distribution is known to us, we have succeeded in finding a standard path integral density leading to unambiguous expectation values for gauge invariant quantitites. Eventually we can compare it with the path integral density derived by conventional techniques, to name the Faddeev-Popov procedure. 
The idea for our procedure is to start from the class of well converging stochastic processes (6.15) and choose in a self-consistent way such specific $\beta_{k}$ and $\gamma$ that the kernel $\widetilde{G}$ appears in the Fokker-Planck operators in just factorized form. This corresponds to a situation where the fluctuation-dissipation theorem applies, which guarantees that in the equilibrium limit the stochastic process becomes independent of $\widetilde{G}$. We will see that the equilibrium distribution of our specially choosen stochastic process is proportional to the exponent of the gauge fixed total action $S_{\text {tot }}$.

To become more specific we recall (6.17), where the adjoint Fokker-Planck operator $L^{\dagger}$ was written out in detail. Suppose that we find a solution for $\beta_{k}$ and $\gamma$ such that

$$
\widetilde{G}^{\mu \nu}\left(t_{1}, t_{2}\right)=\int_{\mathbf{R}} d t_{3}\left[E_{k}^{\mu}\left(t_{1}, t_{3}\right)+\delta_{4}^{\mu} \beta_{k}\left(t_{1}, t_{3}\right)\right] \delta^{k \ell}\left[E_{\ell}^{\nu}\left(t_{2}, t_{3}\right)+\delta^{\nu}{ }_{4} \beta_{\ell}\left(t_{2}, t_{3}\right)\right]
$$

then indeed $\widetilde{G}$ is appearing just in factorized form and we can simplify drastically the adjoint Fokker-Planck operator

$$
L^{\dagger}[\Psi]=\int_{\mathbf{R}^{2}} d t_{1} d t_{2}\left[-\frac{\delta S_{\text {tot }}}{\delta \Psi^{\nu}\left(t_{1}\right)}+\frac{\delta}{\delta \Psi^{\nu}\left(t_{1}\right)}\right] \widetilde{G}^{\nu \mu}\left(t_{1}, t_{2}\right) \frac{\delta}{\delta \Psi^{\mu}\left(t_{2}\right)}
$$

as well as the Fokker-Planck operator itself

$$
L[\Psi]=\int_{\mathbf{R}^{2}} d t_{1} d t_{2} \frac{\delta}{\delta \Psi^{\mu}\left(t_{1}\right)} \widetilde{G}^{\mu \nu}\left[\frac{\delta S_{\mathrm{tot}}}{\delta \Psi^{\nu}\left(t_{2}\right)}+\frac{\delta}{\delta \Psi^{\nu}\left(t_{2}\right)}\right] .
$$

It is not difficult to give a solution for $\beta_{k}$ and $\gamma$ which fulfills (7.1). First we deduce from (6.13) the conditions

$$
\int_{\mathbf{R}} d t_{3} E_{k}^{\bar{\mu}}\left(t_{1}, t_{3}\right) \delta^{k \ell}\left[E_{\ell}^{4}\left(t_{2}, t_{3}\right)+\beta_{\ell}\left(t_{2}, t_{3}\right)\right]=0 \quad \bar{\mu}=1,2,3
$$

as well as

$$
\int_{\mathbf{R}} d t_{3}\left[E^{4}{ }_{k}\left(t_{1}, t_{3}\right)+\beta_{k}\left(t_{1}, t_{3}\right)\right] \delta^{k \ell}\left[E_{\ell}^{4}\left(t_{2}, t_{3}\right)+\beta_{\ell}\left(t_{2}, t_{3}\right)\right]=\gamma\left(t_{1}, t_{2}\right)
$$

and easily find the self-consistent solutions

$$
\beta_{k}\left(t_{1}, t_{2}\right)=-E_{k}^{4}\left(t_{1}, t_{2}\right)+\delta_{k \ell} e^{\ell}{ }_{4}\left(t_{2}, t_{1}\right)
$$

and

$$
\gamma\left(t_{1}, t_{2}\right)=\int_{\mathbf{R}} d t_{3} e^{k}{ }_{4}\left(t_{3}, t_{1}\right) \delta_{k \ell} e^{\ell}{ }_{4}\left(t_{3}, t_{2}\right)
$$


In the last section we claimed that $\widetilde{G}$ could be interpreted as a metric. From (7.1) and from (7.6-7.7) we see that this is indeed the case: We introduce the vielbein $\widetilde{E}$

$$
\widetilde{E}_{k}^{\mu}\left(t_{1}, t_{2}\right)=E^{\mu}{ }_{k}\left(t_{1}, t_{2}\right)+\delta^{\mu}{ }_{4}\left[-E_{k}^{4}\left(t_{1}, t_{2}\right)+\delta_{k \ell} e^{\ell}{ }_{4}\left(t_{2}, t_{1}\right)\right]
$$

with the help of which $\widetilde{G}$ is explicitly decomposable as

$$
\widetilde{G}^{\mu \nu}\left(t_{1}, t_{2}\right)=\int_{\mathbf{R}} d t_{3} \widetilde{E}_{k}^{\mu}\left(t_{1}, t_{3}\right) \delta^{k \ell} \widetilde{E}_{\ell}^{\nu}\left(t_{2}, t_{3}\right)
$$

and by construction is a positive matrix.

We would like to remark, however, that the vielbeins $\widetilde{E}$ are anholonomic as in specific

$$
\frac{\delta \widetilde{E}^{\mu}{ }_{i}\left(t_{1}, t_{2}\right)}{\delta \Phi^{k}\left(t_{3}\right)}-\frac{\delta \widetilde{E}^{\mu}{ }_{k}\left(t_{1}, t_{2}\right)}{\delta \Phi^{i}\left(t_{3}\right)}=\delta^{\mu}{ }_{4}\left[\delta_{i \ell} \frac{\delta e^{\ell}{ }_{4}\left(t_{2}, t_{1}\right)}{\delta \Phi^{k}\left(t_{3}\right)}-\delta_{k \ell} \frac{\delta e^{\ell}{ }_{4}\left(t_{2}, t_{1}\right)}{\delta \Phi^{i}\left(t_{3}\right)}\right] \neq 0
$$

so that there does not exist a coordinate sytem with respect to which the vielbeins $\widetilde{E}$ could be interpreted as the jacobians.

After this discussion of the self-consistent solutions (7.6-7) for $\gamma$ and $\beta_{k}$ which lead to factorized Fokker-Planck operators we now are interested in writing down the corresponding Langevin equations. They read

$$
\begin{aligned}
d \Psi^{\mu}(t, s)= & \int_{\mathbf{R}} d t_{1}\left\{\left[-\widetilde{G}^{\mu \nu}\left(t, t_{1}\right) \frac{\delta S_{\mathrm{tot}}}{\delta \Psi^{\nu}\left(t_{1}\right)}+\frac{\delta \widetilde{G}^{\nu \mu}\left(t, t_{1}\right)}{\delta \Psi^{\nu}\left(t_{1}\right)}\right] d s\right. \\
& \left.+\widetilde{E}^{\mu}{ }_{k}\left(t, t_{1}\right) d W^{k}\left(t_{1}, s\right)\right\}\left.\right|_{\Psi(\cdot)=\Psi(\cdot, s)}
\end{aligned}
$$

and are of a similar form as discussed at the end of section 3). Like in the case of (6.7) the vielbein $\widetilde{E}$ factorizes in a gauge invariant part and a gauge dependent rotation matrix (which could in principle be absorbed, due to the previously mentioned properties of Ito-Langevin equations).

We now derive the equilibrium distribution of the stochastic process described by the above Langevin equation or by the Fokker-Planck equation with Fokker-Planck operator given by (7.3). We remind that we restricted ourselves to the class of well converging stochastic processes, see (6.15) as well as (7.6-6.7), so that the Fokker-Planck probability distribution is normalizable. Most crucially we have that $\widetilde{G}$ is positive, see (7.9); it is appearing in the Fokker-Planck operator in factorized form, see (7.3). As a consequence 
the formal stationary limit of the Fokker- Planck probability distribution can be identified with the equilibrium limit and reads

$$
\rho[\Psi]_{\text {equil. }}=\frac{e^{-S_{\text {tot }}}}{\int D \Psi e^{-S_{\text {tot }}}} .
$$

Now we use the explicit forms of the total actions to explicitly present our result for the path integral density.

In this paper we discussed two specific gauge fixing surfaces; for the case of the variables $\Psi_{\left(\sigma_{1}\right)}$ corresponding to the gauge fixing surface $\varphi^{3}=0$, after integrating out $\Psi_{\left(\sigma_{1}\right)}^{3}$ and $\Psi_{\left(\sigma_{1}\right)}^{4}$, our path integral density obtains

$$
\operatorname{det}\left(1+\left(\Psi_{\left(\sigma_{1}\right)}^{1}\right)^{2}+\left(\Psi_{\left(\sigma_{1}\right)}^{2}\right)^{2}\right)^{-1 / 2} e^{-S_{\mathrm{eff}}\left[\Psi_{\left(\sigma_{1}\right)}^{1}, \Psi_{\left(\sigma_{1}\right)}^{2}\right]} .
$$

Here the effective action in terms of $\Psi^{1}$ and $\Psi^{2}$ is given by

$$
\begin{aligned}
& S_{\text {eff }}\left[\Psi_{\left(\sigma_{1}\right)}^{1}, \Psi_{\left(\sigma_{1}\right)}^{2}\right]=\int d t \frac{1}{2} . \\
& \quad\left[\left(\dot{\Psi}_{\left(\sigma_{1}\right)}^{1}, \dot{\Psi}_{\left(\sigma_{1}\right)}^{2}\right)\left(\begin{array}{cc}
1-\frac{\left(\Psi_{\left(\sigma_{1}\right)}^{2}\right)^{2}}{1+\left(\Psi_{\left(\sigma_{1}\right)}^{1}\right)^{2}+\left(\Psi_{\left(\sigma_{1}\right)}^{2}\right)^{2}} & \frac{\Psi_{\left(\sigma_{1}\right)}^{1} \Psi_{\left(\sigma_{1}\right)}^{2}}{1+\left(\Psi_{\left(\sigma_{1}\right)}^{1}\right)^{2}+\left(\Psi_{\left(\sigma_{1}\right)}^{2}\right)^{2}} \\
\frac{\Psi_{\left(\sigma_{1}\right)}^{1} \Psi_{\left(\sigma_{1}\right)}^{2}}{1+\left(\Psi_{\left(\sigma_{1}\right)}^{1}\right)^{2}+\left(\Psi_{\left(\sigma_{1}\right)}^{2}\right)^{2}} & 1-\frac{\left(\Psi_{\left(\sigma_{1}\right)}^{1}\right)^{2}}{1+\left(\Psi_{\left(\sigma_{1}\right)}^{1}\right)^{2}+\left(\Psi_{\left(\sigma_{1}\right)}^{2}\right)^{2}}
\end{array}\right)\left(\begin{array}{c}
\dot{\Psi}_{\left(\sigma_{1}\right)}^{1} \\
\dot{\Psi}_{\left(\sigma_{1}\right)}^{2}
\end{array}\right)\right. \\
& \left.\quad+\left(\Psi_{\left(\sigma_{1}\right)}^{1}\right)^{2}+\left(\Psi_{\left(\sigma_{1}\right)}^{2}\right)^{2}\right]
\end{aligned}
$$

and is agreeing nicely with the result of [13].

For the coordinates $\Psi_{\left(\sigma_{2}\right)}$ the integration over $\Psi_{\left(\sigma_{2}\right)}^{3}$ and $\Psi_{\left(\sigma_{2}\right)}^{4}$ does not leave any trace and the path integral measure is just the exponent of the extremely simple effective action

$$
S_{\mathrm{eff}}\left[\Psi_{\left(\sigma_{2}\right)}^{1}, \Psi_{\left(\sigma_{2}\right)}^{2}\right]=\int d t \frac{1}{2}\left[\left(\dot{\Psi}_{\left(\sigma_{2}\right)}^{1}(t)\right)^{2}+\left(\dot{\Psi}_{\left(\sigma_{2}\right)}^{2}(t)\right)^{2}+\left(\Psi_{\left(\sigma_{2}\right)}^{1}(t)\right)^{2}+\left(\Psi_{\left(\sigma_{2}\right)}^{2}(t)\right)^{2}\right] .
$$

It is evident that this gauge fixing leads to a quadratic action and it is therefore completely trivial to calculate the only relevant gauge invariant quantity, the two point function

$$
\lim _{s \rightarrow \infty}\left\langle\left(\Psi_{\left(\sigma_{2}\right)}^{1}(t)\right)^{2}+\left(\Psi_{\left(\sigma_{2}\right)}^{2}(t)\right)^{2}\right\rangle(s)=2 \pi
$$

It nicely agrees with the corresponding result in Dirac's scheme (see e.g. [13] and [14]) or with the path integral results in [13]. 
At the end of this section we transform the final Langevin equation (7.11) back into the original cartesian coordinates $\Phi^{k}$. Amusingly we end up with a Langevin equation with a similar structure as in (7.11): First we have with the Ito rules

$$
\begin{aligned}
d \Phi^{i}(t, s)= & \int_{\mathbf{R}} d t_{1}\left[e^{i}{ }_{\mu}\left(t, t_{1}\right) d \Psi^{\mu}\left(t_{1}\right)\right. \\
& \left.+\int_{\mathbf{R}} d t_{2} \frac{\delta e^{i}{ }_{\mu}\left(t, t_{1}\right)}{\delta \Psi^{\nu}\left(t_{2}\right)} \widetilde{G}^{\mu \nu}\left(t_{1}, t_{2}\right)\right]\left.\right|_{\Psi(\cdot)=\Psi(\cdot, s)} .
\end{aligned}
$$

Due to the fact that the $\Phi$ are flat coordinates we have - similarly as (3.9) -

$$
\int d t_{1} \frac{\delta e^{k}{ }_{\mu}\left(t_{1}, t_{2}\right)}{\delta \Phi^{k}\left(t_{1}\right)}=0
$$

so that we finally arrive at

$$
\begin{aligned}
d \Phi^{i}(t, s)= & \int_{\mathbf{R}} d t_{1}\left\{\left[-\hat{g}^{i k}\left(t, t_{1}\right) \frac{\delta S_{\text {tot }}}{\delta \Phi^{k}\left(t_{1}\right)}+\frac{\delta \hat{g}^{k i}\left(t_{1}, t\right)}{\delta \Phi^{k}\left(t_{1}\right)}\right] d s\right. \\
& \left.+\hat{e}_{k}^{i}\left(t, t_{1}\right) d W^{k}\left(t_{1}, s\right)\right\}\left.\right|_{\Phi(\cdot)=\Phi(\cdot, s)} .
\end{aligned}
$$

Here we defined new vielbeins $\hat{e}$

$$
\hat{e}_{k}^{i}\left(t_{1}, t_{2}\right)=\delta_{k}^{i} \delta\left(t_{1}-t_{2}\right)+\int_{\mathbf{R}} d t_{3} e_{4}^{i}\left(t_{1}, t_{3}\right)\left[-E_{k}^{4}\left(t_{3}, t_{2}\right)+\delta_{k \ell} e_{4}^{\ell}\left(t_{2}, t_{3}\right)\right]
$$

and a metric $\hat{g}$

$$
\hat{g}^{i k}\left(t_{1}, t_{2}\right)=\int_{\mathbf{R}} d t_{3} \hat{e}_{\ell}^{i}\left(t_{1}, t_{3}\right) \delta^{\ell m} \hat{e}_{m}^{k}\left(t_{2}, t_{3}\right) .
$$

We recognize that the Langevin equation (7.20) indeed has the same form as (7.11); the same conclusions apply about the application of the fluctuation dissipation theorem and the independence of the stochastic process of $\hat{g}$ in the equilibrium limit.

We close with the remark that although the Langevin equation (7.20) is defined with respect to the original cartesian field coordinates the procedure of stochastic gauge fixing has introduced a dependence of the new coordinates $\Psi_{(\sigma)}$.

\section{Geometrical aspects of the generalized stochastic gauge fixing procedure}

The main virtue of the generalized stochastic gauge fixing procedure lies in the fact that for specific choices of the extra functions $\alpha$ and $\beta_{k}$ the fluctuation-dissipation theorem 
can be applied; as a consequence the equilibrium distribution can be derived quite easily. Thereby we were led to introduce in (6.13) a positive definite, matrix-valued kernel $\widetilde{G}^{\mu \nu}$ which can be regarded as the inverse of a particular matrix, denoted by $\widetilde{G}_{\mu \nu}$, on $\Sigma_{(\sigma)} \times \mathcal{G}$. What can now be said about its geometrical meaning?

We will begin with the explicit calculation of $\widetilde{G}_{\mu \nu}$. First it can be shown by a direct calculation that the inverse vielbein of $\widetilde{E}^{\mu}{ }_{k}$ which we denote by $\widetilde{e}_{\mu}^{i}$ is given by

$$
\begin{aligned}
\widetilde{e}_{\mu}^{i}\left(t, t^{\prime}\right)= & e_{\mu}^{i}\left(t, t^{\prime}\right)+\int_{\mathbf{R}^{3}} d t_{1} d t_{2} d t_{3} Z^{i}\left(t, t_{1}\right) K^{-1}\left(t_{1}, t_{2}\right) \\
& {\left[E_{j}^{4}\left(t_{2}, t_{3}\right)-\delta_{j k} Z^{k}\left(t_{3}, t_{2}\right)\right] e^{j}{ }_{\mu}\left(t_{3}, t^{\prime}\right) . }
\end{aligned}
$$

Here the kernel $K^{-1} \in C^{\infty}\left(\mathbf{R}^{2}, \mathbf{R}\right)$ is uniquely defined by

$$
\int_{\mathbf{R}} d t^{\prime} K^{-1}\left(t_{1}, t^{\prime}\right) G_{44}\left(t^{\prime}, t_{2}\right)=\delta\left(t_{1}-t_{2}\right)
$$

where $G_{\mu \nu}$ denotes the metric inverse to $G^{\mu \nu}$,

$$
G_{\mu \nu}\left(\tau_{1}, \tau_{2}\right)=\int_{\mathbf{R}} d t e^{i}{ }_{\mu}\left(t, \tau_{1}\right) \delta_{i j} e^{j}{ }_{\nu}\left(t, \tau_{2}\right)
$$

and the following identities hold

$$
\begin{aligned}
\int_{\mathbf{R}} d t \widetilde{e}^{k}{ }_{\mu}\left(t_{1}, t\right) \widetilde{E}^{\mu}{ }_{m}\left(t, t_{2}\right) & =\delta^{k}{ }_{m} \delta\left(t_{1}-t_{2}\right) \\
\int_{\mathbf{R}} d t \widetilde{e}^{k}{ }_{\mu}\left(t_{1}, t\right) \widetilde{E}^{\nu}{ }_{k}\left(t, t_{2}\right) & =\delta_{\mu}{ }^{\nu} \delta\left(t_{1}-t_{2}\right) .
\end{aligned}
$$

The components of the metric $\widetilde{G}=\int_{\mathbf{R}^{2}} d \tau_{1} d \tau_{2} \widetilde{G}_{\mu \nu}\left(\tau_{1}, \tau_{2}\right) \delta \Psi^{\mu}\left(\tau_{1}\right) \otimes \delta \Psi^{\nu}\left(\tau_{2}\right)$ are then given by

$$
\begin{aligned}
\widetilde{G}_{\mu \nu}\left(\tau_{1}, \tau_{2}\right) & =\int_{\mathbf{R}} d t \widetilde{e}^{i}{ }_{\mu}\left(t, \tau_{1}\right) \delta_{i j} \widetilde{e}^{j}{ }_{\nu}\left(t, \tau_{2}\right) \\
& =\int_{\mathbf{R}^{2}} d \tau d \tau^{\prime} \int_{\mathbf{R}} d t \widetilde{e}^{i}{ }_{\mu}\left(t, \tau_{1}\right) E^{\lambda_{1}}{ }_{i}(\tau, t) G_{\lambda_{1} \lambda_{2}}\left(\tau, \tau^{\prime}\right) \int_{\mathbf{R}} d t^{\prime} E^{\lambda_{2}}{ }_{j}\left(\tau^{\prime}, t^{\prime}\right) \widetilde{e}^{j}{ }_{\nu}\left(t^{\prime}, \tau_{2}\right) \\
& =\widehat{G}_{\mu \nu}\left(\tau_{1}, \tau_{2}\right)+\delta_{\mu}{ }^{4} \delta^{4}{ }_{\nu} K^{-1}\left(\tau_{1}, \tau_{2}\right) .
\end{aligned}
$$

In the above equation we introduced

$$
\widehat{G}_{\mu \nu}\left(\tau_{1}, \tau_{2}\right)=G_{\mu \nu}\left(\tau_{1}, \tau_{2}\right)-\int_{\mathbf{R}^{2}} d t d t^{\prime} G_{\mu 4}\left(\tau_{1}, t\right) K^{-1}\left(t, t^{\prime}\right) G_{4 \nu}\left(t^{\prime}, \tau_{2}\right)
$$


From a more geometrical viewpoint, the matrix valued kernel $\int_{\mathbf{R}} d t E^{\nu}{ }_{i}\left(\tau_{1}, t\right) \widetilde{e}_{\mu}^{i}\left(t, \tau_{2}\right)$ in $(8.5)$ can be regarded as component of a vector valued one form $\varepsilon$ on $\Sigma_{(\sigma)} \times \mathcal{G}$, namely

$$
\varepsilon=\int_{\mathbf{R}^{3}} d t_{1} d t_{2} d t_{3} E^{\nu}{ }_{i}\left(t_{1}, t_{2}\right) \tilde{e}_{\mu}^{i}\left(t_{2}, t_{3}\right) \delta \Psi^{\mu}\left(t_{3}\right) \otimes \frac{\delta}{\delta \Psi^{\nu}\left(t_{1}\right)} .
$$

Insertion of (8.1) yields

$$
\begin{aligned}
\varepsilon= & \int_{\mathbf{R}} d t \delta \Psi^{\mu}(t) \otimes \frac{\delta}{\delta \Psi^{\mu}(t)}-\int_{\mathbf{R}^{3}} d t_{1} d t_{2} d t_{3} K^{-1}\left(t_{1}, t_{2}\right) G_{4 \mu}\left(t_{2}, t_{3}\right) \delta \Psi^{\mu}\left(t_{3}\right) \otimes \frac{\delta}{\delta \Psi^{4}\left(t_{1}\right)} \\
& +\int_{\mathbf{R}^{2}} d t_{1} d t_{2} K^{-1}\left(t_{1}, t_{2}\right) \delta \Psi^{4}\left(t_{2}\right) \otimes \frac{\delta}{\delta \Psi^{4}\left(t_{1}\right)}
\end{aligned}
$$

so that for each vector field $X$ on $\Sigma_{(\sigma)} \times \mathcal{G}$ we finally get the formula

$$
\varepsilon(X)=X-Z_{B(X)}+Z_{\Theta(X)}
$$

Here both

$$
B(\tau)=\int_{\mathbf{R}^{2}} d t_{1} d t_{2} K^{-1}\left(\tau, t_{1}\right) G_{4 \mu}\left(t_{1}, t_{2}\right) \delta \Psi^{\mu}\left(t_{2}\right)
$$

and

$$
\Theta(\tau)=\int_{\mathbf{R}} d t_{1} K^{-1}\left(\tau, t_{1}\right) \delta \Psi^{4}\left(t_{1}\right)
$$

are Lie $\mathcal{G}$-valued one forms on $\Sigma_{(\sigma)} \times \mathcal{G}$. Since the form $B$ is $\mathcal{G}$-invariant and $B\left(Z_{\xi}\right)=\xi$ $\forall \xi \in$ Lie $\mathcal{G}$, it defines a connection in the trivial principal $\mathcal{G}$-bundle $\Sigma_{(\sigma)} \times \mathcal{G} \rightarrow \Sigma_{(\sigma)}$. However, the corresponding horizontal subbundle, which is given by all those vector fields $X$ on $\Sigma_{(\sigma)} \times \mathcal{G}$ which are annihilated by $B$, i.e. $B(X)=0$, is orthogonal to the gauge orbits with respect to the original metric $G_{\mu \nu}$. This can be proven by a direct calculation.

Hence the term $X-Z_{B(X)}$ in (8.9) precisely gives the projection of $X$ onto this horizontal subbundle. In summary, the global form of $\widetilde{G}$ is given by

$$
\widetilde{G}(X, Y)=G(\varepsilon(X), \varepsilon(Y))=G\left(X-Z_{B(X)}, Y-Z_{B(Y)}\right)+G\left(Z_{\Theta(X)}, Z_{\Theta(Y)}\right)
$$

for any two vector fields $X, Y$ on $\Sigma_{(\sigma)} \times \mathcal{G}$. From our discussion it is evident that the terms appearing in the second part of equation (8.12) correspond exactly to $\widehat{G}_{\mu \nu}\left(\tau_{1}, \tau_{2}\right)$ and $\delta_{\mu 4} \delta_{\nu 4} K^{-1}\left(\tau_{1}, \tau_{2}\right)$ of (8.4) respectively. Hence $\widehat{G}_{\mu \nu}$ is the induced metric on the horizontal subbundle, which descends to a well-defined metric on $\Sigma_{(\sigma)}$. In fact, for $\bar{\mu}, \bar{\nu}=1,2,3$ this 
horizontal metric $\widehat{G}_{\bar{\mu} \bar{\nu}}$ can be shown to be the inverse of the kernel $G^{\bar{\mu} \bar{\nu}}$ appearing in the Langevin equations (6.7) of the gauge invariant variables.

Before going on, however, we recall that due to the result (7.10), no coordinate system exists such that $\varepsilon$ could be interpreted as a jacobian.

The curvature $\mathcal{F}$ of the connection is given by

$$
\mathcal{F}=\frac{1}{2} \int_{\mathbf{R}^{2}} d t_{1} d t_{2}\left[\frac{\delta B_{\mu}\left(t_{1}\right)}{\delta \Psi^{\nu}\left(t_{2}\right)}-\frac{\delta B_{\nu}\left(t_{2}\right)}{\delta \Psi^{\mu}\left(t_{1}\right)}\right] \delta \Psi^{\mu}\left(t_{1}\right) \wedge \delta \Psi^{\nu}\left(t_{2}\right) .
$$

An explicit calculation yields that it is nonvanishing. Hence there does not exist a manifold whose tangent bundle would coincide with the horizontal subbundle defined by the connection $B$.

At the end we would like to give some comments on the geometrical meaning of (7.14) and (7.15). The action $\mathcal{R}$ in (4.2) induces a free action $\hat{\mathcal{R}}$ on $\mathcal{E}$

$$
\hat{\mathcal{R}}(\vec{\varphi}, g)(t)=\left(R(g(t)) \underline{\varphi}(t), \varphi^{3}(t)-g(t)\right),
$$

in a natural way. As a consequence, the projection $\hat{\pi}: \mathcal{E} \rightarrow \mathcal{M}:=\mathcal{E} / \mathcal{G}$, with $\hat{\pi}(\vec{\varphi})=[\vec{\varphi}]_{\mathcal{M}}$, where $[\vec{\varphi}]_{\mathcal{M}}$ denotes the equivalence class of $\vec{\varphi}$ with respect to the action $\hat{\mathcal{R}}$, also admits the structure of a principal $\mathcal{G}$-bundle over $\mathcal{M}$, where the quotient is taken with respect to $\hat{\mathcal{R}}$. Moreover, $\mathcal{E} \times_{\mathcal{G}} \mathcal{A}$ can be regarded as an associated fiber bundle over $\mathcal{M}$ with typical fiber $\mathcal{A}$ and projection $\tilde{\pi}(\vec{\varphi}, A)=\hat{\pi}(\vec{\varphi})=[\vec{\varphi}]_{\mathcal{M}}$. The section $\sigma_{1}$ which was defined in (4.4) induces a global section $\hat{\sigma}_{1}$ of $\hat{\pi}: \mathcal{E} \rightarrow \mathcal{M}$ by $\hat{\sigma}_{1}\left([\vec{\varphi}]_{\mathcal{M}}\right)=\left(R\left(\varphi^{3}\right) \underline{\varphi}, 0\right)$. Let $\hat{\Sigma}_{\left(\sigma_{1}\right)}=\operatorname{im} \hat{\sigma}_{1}$ denote the corresponding gauge fixing surface in $\mathcal{E}$, then the projection $\Sigma_{\left(\sigma_{1}\right)} \rightarrow \hat{\Sigma}_{\left(\sigma_{1}\right)}$ yields a fiber bundle with typical fiber $\mathcal{A}$ equivalent to $\tilde{\pi}: \mathcal{E} \times_{\mathcal{G}} \mathcal{A} \rightarrow \mathcal{M}$. Integrating out $\Psi_{\left(\sigma_{1}\right)}^{4}$ corresponds to fiber integration along the gauge group in the principal bundle $\mathcal{E} \times \mathcal{A} \rightarrow \Sigma_{\left(\sigma_{1}\right)}$ and integrating out $\Psi_{\left(\sigma_{1}\right)}^{3}$ corresponds to fiber integration along the fiber $\mathcal{A}$ in $\Sigma_{\left(\sigma_{1}\right)} \rightarrow \hat{\Sigma}_{\left(\sigma_{1}\right)}$

The geometrical interpretation of (7.15) is slightly different. The action $\mathcal{R}$ in (4.2) also yields an induced action of $\mathcal{G}$ on $\mathcal{A}$, which although it is not free, also [41, 42] gives rise to a smooth quotient $\mathcal{N}:=\mathcal{A} / \mathcal{G}$. Since every gauge potential $A \in \mathcal{A}$ can be gauged away by a suitable gauge transformation, the orbit space $\mathcal{N}$ consists only of the point [0]. 
Like in the former case, $\mathcal{E} \times_{\mathcal{G}} \mathcal{A}$ can be regarded as a fiber bundle over $\mathcal{N}=\{[0]\}$ with typical fiber $\mathcal{E}$. Integrating out $\Psi_{\left(\sigma_{2}\right)}^{4}$ now corresponds to fiber integration along $\mathcal{G}$ in the principal bundle $\mathcal{E} \times \mathcal{A} \rightarrow \Sigma_{\left(\sigma_{2}\right)}$. Since $\Sigma_{\left(\sigma_{2}\right)} \simeq\{0\} \times \mathcal{E}$, integrating out $\Psi_{\left(\sigma_{2}\right)}^{i}, i=1,2,3$, corresponds purely to an integration over $\mathcal{E}$.

\section{Outlook}

In this paper we performed the stochastic quantization of the helix model and were able to show agreement with the path integral procedure in a nonperturbative way.

We generalized the stochastic gauge fixing procedure by not only introducing an extra drift term horizontally to the gauge orbits, but by also modifying the Wiener process itself. This modification was done in such a way that expectation values of gauge invariant objects remained unchanged. We gave a detailed analysis of the underlying geometrical structures of the model. Although the newly introduced extra terms at first sight seemed to render our approach complicated, just the contrary was proven to be the case: Due to a delicate interplay between the total drift term and the modified diffusion term the fluctuation dissipation theorem could be applied and the equilibrium limit of the stochastic process was derivable in a straightforward manner.

As one out of several immediate applications of our nonperturbative stochastic approach we are presently studying the helix model for the case where gauge fixing surfaces can be defined only locally; another work in progress is the phase space formulation of the helix model and the introduction of the BRST scheme.

\section{Acknowledgement}

We thank H. Grosse, H. Nakazato and K. Okano for valuable discussions. H. H. is grateful for the kind hospitality offered to him by the High Energy Physics Group of Tokuyama University, Tokuyama and by the High Energy Physics Group of the Dept. of Physics of Waseda University, Tokyo, where this paper was completed; he is thankful for financial 
support from the Japanese Society of the Promotion of Science.

\section{Appendix}

For the case of the variables $\Psi_{\left(\sigma_{1}\right)}($ see $(4.15)-(4.16))$ the vielbeins $E$ and $e$ (see (3.4)) are given by, respectively

$$
\begin{aligned}
E_{i}^{\mu}\left(t, t^{\prime}\right) & =\left(\begin{array}{cccc}
\cos \varphi^{3}(t) & -\sin \varphi^{3}(t) & -\Psi^{2}(t) & 0 \\
\sin \varphi^{3}(t) & \cos \varphi^{3}(t) & \Psi^{1}(t) & 0 \\
0 & 0 & -\partial_{t} & 1 \\
0 & 0 & -1 & 0
\end{array}\right) \delta\left(t-t^{\prime}\right) \\
e^{i}{ }_{\mu}\left(t, t^{\prime}\right) & =\left(\begin{array}{cccc}
\cos \varphi^{3}(t) & \sin \varphi^{3}(t) & 0 & -\varphi^{2}(t) \\
-\sin \varphi^{3}(t) & \cos \varphi^{3}(t) & 0 & \varphi^{1}(t) \\
0 & 0 & 0 & -1 \\
0 & 0 & 1 & -\partial_{t}
\end{array}\right) \delta\left(t-t^{\prime}\right)
\end{aligned}
$$

Furthermore we have

$$
E^{\mu}{ }_{i}\left(t, t^{\prime}\right)=\left(\begin{array}{cccc}
1 & 0 & -\Psi^{2}(t) & 0 \\
0 & 1 & \Psi^{1}(t) & 0 \\
0 & 0 & -\partial_{t} & 1 \\
0 & 0 & -1 & 0
\end{array}\right)\left(\begin{array}{cccc}
\cos \varphi^{3}(t) & -\sin \varphi^{3}(t) & 0 & 0 \\
\sin \varphi^{3}(t) & \cos \varphi^{3}(t) & 0 & 0 \\
0 & 0 & 1 & 0 \\
0 & 0 & 0 & 1
\end{array}\right) \delta\left(t-t^{\prime}\right)
$$

In the case of the variables $\Psi_{\left(\sigma_{2}\right)}($ see $(4.17)-(4.18),(4.6))$ we have

$$
E^{\mu}{ }_{i}\left(t, t^{\prime}\right)=\left(\begin{array}{cccc}
\cos F(t) \delta\left(t-t^{\prime}\right) & -\sin F(t) \delta\left(t-t^{\prime}\right) & -\Psi^{2}(t) h\left(t^{\prime}\right) & -\Psi^{2}(t) \Xi\left(t, t^{\prime}\right) \\
\sin F(t) \delta\left(t-t^{\prime}\right) & \cos F(t) \delta\left(t-t^{\prime}\right) & \Psi^{1}(t) h\left(t^{\prime}\right) & \Psi^{1}(t) \Xi\left(t, t^{\prime}\right) \\
0 & 0 & \delta\left(t-t^{\prime}\right)-h\left(t^{\prime}\right) & -\Xi\left(t, t^{\prime}\right) \\
0 & 0 & -h\left(t^{\prime}\right) & -\Xi\left(t, t^{\prime}\right)
\end{array}\right)
$$

where

$$
\Xi\left(t, t^{\prime}\right)=\Theta\left(t-t^{\prime}\right)-\int_{t^{\prime}}^{\infty} d \tau h(\tau)
$$


Furthermore

$$
e_{\mu}^{i}\left(t, t^{\prime}\right)=\left(\begin{array}{cccc}
\cos F(t) \delta\left(t-t^{\prime}\right) & \sin F(t) \delta\left(t-t^{\prime}\right) & 0 & -\varphi^{2}(t) \delta\left(t-t^{\prime}\right) \\
-\sin F(t) \delta\left(t-t^{\prime}\right) & \cos F(t) \delta\left(t-t^{\prime}\right) & 0 & \varphi^{1}(t) \delta\left(t-t^{\prime}\right) \\
0 & 0 & \delta\left(t-t^{\prime}\right)-h(t) h\left(t^{\prime}\right) & -\delta\left(t-t^{\prime}\right) \\
0 & 0 & 0 & -\partial_{t} \delta\left(t-t^{\prime}\right)
\end{array}\right)
$$

as well as

$$
\begin{aligned}
E_{i}^{\mu}\left(t, t^{\prime}\right)= & \left(\begin{array}{cccc}
\delta\left(t-t^{\prime}\right) & 0 & -\Psi^{2}(t) h\left(t^{\prime}\right) & -\Psi^{2}(t) \Xi\left(t, t^{\prime}\right) \\
0 & \delta\left(t-t^{\prime}\right) & \Psi^{1}(t) h\left(t^{\prime}\right) & \Psi^{1}(t) \Xi\left(t, t^{\prime}\right) \\
0 & 0 & \delta\left(t-t^{\prime}\right)-h\left(t^{\prime}\right) & -\Xi\left(t, t^{\prime}\right) \\
0 & 0 & -h\left(t^{\prime}\right) & -\Xi\left(t, t^{\prime}\right)
\end{array}\right) \\
& \cdot\left(\begin{array}{cccc}
\cos F(t) & -\sin F(t) & 0 & 0 \\
\sin F(t) & \cos F(t) & 0 & 0 \\
0 & 0 & 1 & 0 \\
0 & 0 & 0 & 1
\end{array}\right) .
\end{aligned}
$$

\section{References}

[1] G. Parisi and Wu Yongshi, Sci.Sin. 24 (1981) 483

[2] P. Damgaard and H. Hüffel, Phys. Rep. 152 (1987) 227

[3] M. Namiki, "Stochastic Quantization", Springer, Heidelberg, 1992

[4] G. Jona-Lasinio and P. Mitter, Commun. Math. Phys. 101 (1983) 343

[5] P. Damgaard, H. Hüffel and A. Rosenblum, "Probabilistic Methods in Quantum Field Theory and Quantum Gravity", Plenum Press, New York, 1990

[6] M. Namiki and K. Okano, Prog. Theor. Phys. 111 (1993) Suppl.

[7] L. Faddeev and V. Popov, Phys. Lett. 25B (1967) 29 
[8] V. Gribov, Nucl. Phys. B139 (1978) 1

[9] I. Singer, Comm. Math. Phys. 60 (1978) 1

[10] D. Zwanziger, Nucl. Phys. B345 (1990) 461

[11] S. Shabanov, Phys. Let. B318 (1993) 323

[12] C. Becchi and C. Imbimbo, Nucl. Phys. B462 (1996) 571

[13] R. Friedberg, T.D. Lee, Y. Pang and H. Ren, Ann. of Phys. 246 (1996) 381

[14] K. Fujikawa, Nucl. Phys. B468 (1996) 355

[15] E. Seiler, Acta Phys. Austriaca, Suppl. XXVI (1984) 259

[16] D. Zwanziger, in "Fundamental Problems of Gauge Field Theory", A. Wightman and G. Velo eds., Plenum, 1986

[17] D. Zwanziger, Nucl. Phys. B192 (1981) 259

[18] S. Marculescu, K. Okano, L. Schülke and B. Zheng, Nucl. Phys. B417 (1994) 579

[19] L. Baulieu and D. Zwanziger, Nucl. Phys. B193 (1981) 163

[20] C. Ordonez, M. Rubin and D. Zwanziger, Phys. Rev. D40 (1989) 4056

[21] M. Gotay, Journ. Math. Phys. 27 (1986) 2051

[22] G. Tuynman, Journ. Math. Phys. 31 (1991) 83

[23] C. Emmrich, Commun. Math. Phys. 151 (1993) 531

[24] J. Hetrick, Int. Journ. of Mod. Phys. A9 (1994) 3153

[25] L. Chandar and E. Ercolessi, Nucl. Phys. B426 (1994) 94

[26] J. Fuchs, M. Schmidt and Ch. Schweigert, Nucl. Phys. B426 (1994) 107 
[27] W. Greub, S. Halperin and R. Vanstone, "Connections, Curvature and Cohomology", Academic Press, New York, 1972

[28] B. DeWitt, in "General Relativity: An Einstein Centenary Survey", S. Hawking and W. Israel (eds.), Cambridge Univ. Press, Cambridge, 1979.

[29] K. Kuchař, Phys. Rev. D34 (1986) 3044; Phys. Rev. D35 (1987) 596.

[30] H. Hüffel, "Stochastic Quantization of Gauge Models", talk given at the Second Meeting on Constrained Dynamics and Quantum Gravity, Santa Margherita Ligure, Italy, September 1996, Univ. of Vienna preprint UWThPh-1997-02

[31] H. Hüffel and G. Kelnhofer, "Generalized Stochastic Gauge Fixing", Univ. of Vienna preprint UWThPh-1997-05

[32] H. Hüffel and G. Kelnhofer, in preparation

[33] L. Arnold, "Stochastische Differentialgleichungen", Oldenbourg, München, 1973

[34] C. Gardiner, "Handbook of Stochastic Methods", Springer, Berlin, 1990

[35] N. Ikeda and S. Watanabe, "Stochastic Differential Equations and Diffusion Processes", North Holland, Amsterdam, 1981

[36] R. Graham, Z. Physik B26 (1977) 397; Phys. Lett. 109A (1985) 209

[37] S. Caracciolo, H.-C. Ren and Y.-S. Wu, Nucl. Phys. B260 (1985) 381

[38] G. Batrouni, H. Kawai, P. Rossi, J. Math. Phys. 27 (1986) 1646.

[39] K. Okano, L. Schülke and B. Zheng, Phys. Lett. B258 (1991) 421

[40] S.Marculescu, K. Okano and L. Schülke, Nucl. Phys. B349 (1991) 463

[41] G. Bredon, "Introduction to Compact Transformation Groups", Academic Press, New York, 1972 
[42] K. Jänich, "Differenzierbare G-Mannigfaltigkeiten", Lecture Notes in Mathematics 59, Springer, 1968 


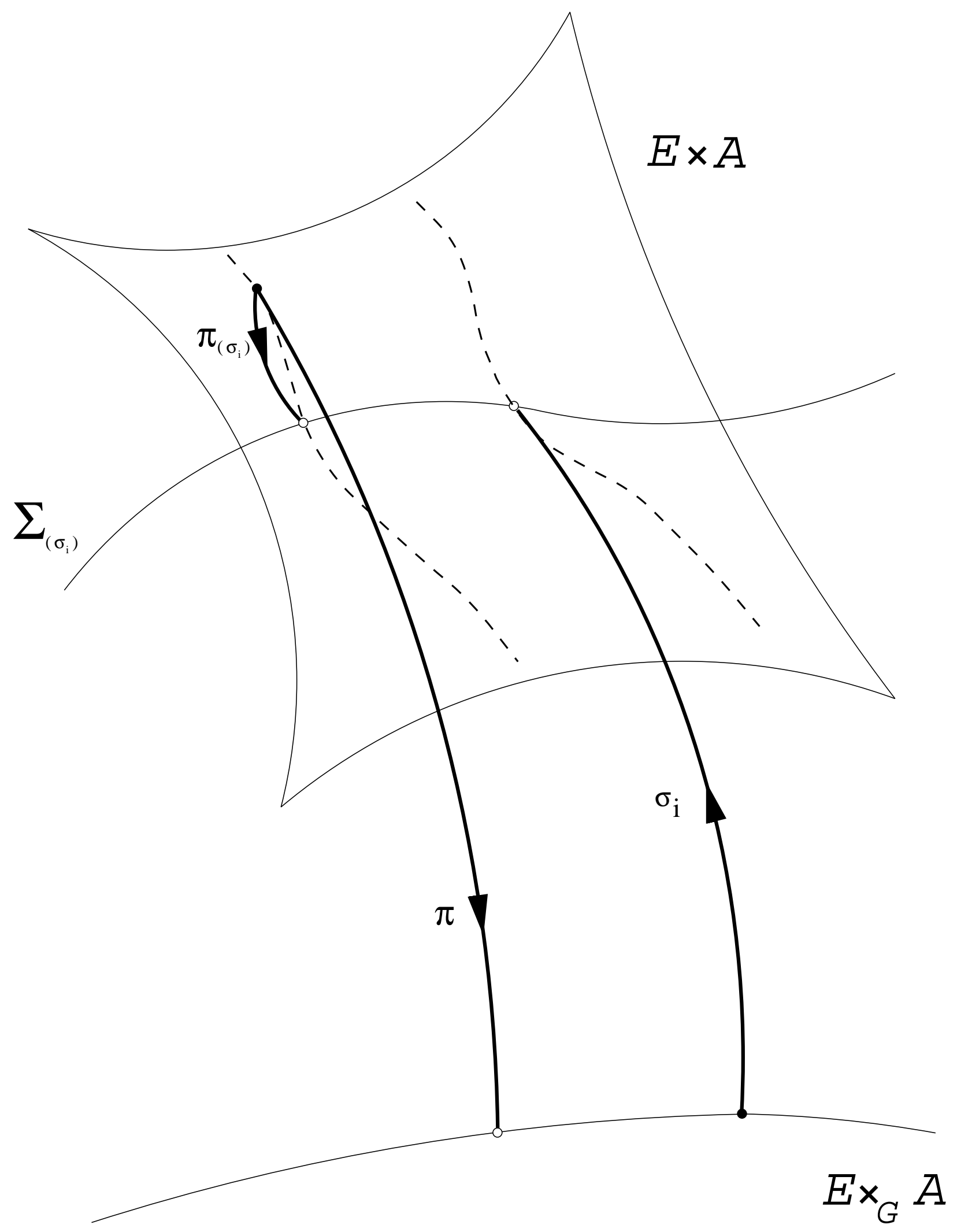

Fig. 1. Geometrical structures 